\title{
The discovery of new and more potent chloropyramine (C4) analogues for the potential treatment of invasive breast cancer
}

\author{
Sahar Kandil", Filippo Prencipe, Samuel Jones, Stephen Hiscox and Andrew D. Westwell \\ School of Pharmacy \& Pharmaceutical Sciences, Cardiff University, King Edward VII Avenue, Cardiff, CF10 3NB, \\ Wales, United Kingdom. \\ Keywords: Focal adhesion kinase (FAK), metastatic breast cancer (BC), chloropyramine (C4), lead optimisation, \\ reductive amination.
}

Graphical abstract

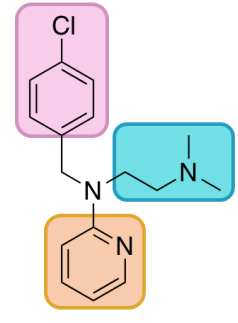

C4 $\left(\mathrm{IC}_{50}=>100 \mu \mathrm{M}\right)$

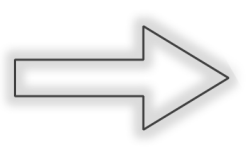

$\mathbf{5 c}\left(\mathrm{IC}_{50}=23.5-31.3 \mu \mathrm{M}\right)$

\section{Highlights}

- 17 New chloropyramine analogues were designed and synthesised.

- All new compounds were evaluated for their activity in three different breast cancer cell lines.

- For the most active compound $\mathbf{5 c}$, in vitro pharmacokinetic studies were performed.

\section{ABSTRACT}

Breast cancer $(B C)$ is the second most common cancer worldwide, accounting for $25 \%$ of all female cancers. Although the survival rate has increased significantly in the past few decades, patients who develop secondary site metastasis as well as those diagnosed with triple negative breast cancer (TNBC) still represent a real unmet medical challenge. Previous studies have shown that chloropyramine (C4) inhibits FAK-VEGFR3 signaling. More recently, C4 is reported to have SASH1 inducing properties. However, C4 exerts its antitumour and antiangiogenic effects at high micromolar concentrations $(>100 \mu \mathrm{M})$ that would not be compatible with further drug development against invasive breast cancer driven by FAK signaling. In this study, molecular modeling guided structural modifications have been introduced to the chloropyramine $\mathbf{C 4}$ scaffold to improve its activity in breast cancer cell lines. Seventeen compounds were designed and synthesised and their anti-proliferative activity was evaluated against three human breast cancer lines (MDA-MB-231, BT474 and T47D). Compound 5c was identified to display an average activity of $\mathrm{IC}_{50}=23.5-31.3 \mu \mathrm{M}$, which represents a significant improvement of $\mathbf{C 4}$ activity in the same assay model. Molecular modelling and pharmacokinetic studies provided more promising insights into the mechanistic features of this new series.

\footnotetext{
* Corresponding author

E-mail address: Kandils1@cardiff.ac.uk (S. Kandil)
} 


\section{INTRODUCTION}

Current therapeutic strategies for breast cancer $(B C)$ are mainly directed towards primary tumour site histopathology i.e. expression of estrogen (ER), progesterone (PR) and human epidermal growth factor 2 (HER2) receptors. Due to improved therapeutic strategies, the survival rate for ER-positive breast cancer in particular has increased significantly in the past few decades. However, the subgroup of aggressive and invasive breast cancers, including triple-negative disease (around $15 \%$ of cases), is characterised by rapid relapse following chemotherapy and progression to metastatic disease, with a poor overall prognosis. ${ }^{1}$

Focal adhesion kinase (FAK) is a cytoplasmic tyrosine kinase and scaffold protein, which is localised to focal adhesions, and forms points of contacts between cells and the extracellular matrix. FAK is highly expressed in cancer, acts as a multifunctional mediator of cell signaling, and its involvement in cancer cell proliferation, invasion and metastasis is well documented..$^{2-7}$ It is overexpressed in many types of cancer such as thyroid, brain, liver, prostate, breast, colon, head and neck. Moreover, this overexpression is associated with an invasive phenotype in such tumours. ${ }^{7}$ Numerous approaches are being developed to inhibit FAK catalytic function (mainly targeting the Y397 tyrosine phosphorylation site), which have successfully led to the development of clinical stage FAK kinase inhibitors such as defactinib, currently in Phase $1 /$ II trials in ovarian, non-small cell lung, mesothelioma and pancreatic cancer (Verastem, Inc.) $)^{8}$ Alternatively, destabilisation of the FAK scaffolding function is believed to be an advantageous and potentially more specific strategy to develop FAK targeting therapeutics rather than focusing on the kinase function only. ${ }^{7-11}$ In silico screening of the National Cancer Institute's small molecule library for compounds targeting the FAKVEGFR3 interactions led to the identification of the clinically approved antihistamine drug chloropyramine (C4) which belongs to the first generation of $\mathrm{H} 1$-antihistamines. ${ }^{12,13}$ Further in vitro and in vivo studies have shown that treatment with $\mathbf{C 4}$ showed reduction in tumour burden and neovascularization in mouse models of breast cancer. ${ }^{12}$ This activity has been demonstrated, both alone and synergistically with other chemotherapeutic agents (e.g. doxorubicin and gemcitabine), sensitising breast cancer cells to chemotherapy. ${ }^{12,13}$ Moreover, C4 is under development by CureFAKtor Pharmaceuticals LLC, and received orphan drug designation from the FDA in combination with gemcitabine for the treatment of pancreatic cancer. ${ }^{14}$ More recently, Burgess et al have independently used in silico connectivity mapping and in vitro modelling to demonstrate that chloropyramine C4, increased SASH1 protein levels in breast cancer cells. ${ }^{15}$ Expression of the SASH1 protein is reduced in a range of human cancers and has been implicated in apoptotic cancer cell death. Although $\mathbf{C 4}$ is an attractive candidate, it exerts its antitumour and antiangiogenic effects at high micromolar concentrations $(>100 \mu \mathrm{M}) .{ }^{13}$ In order to get more insights into the structure activity 
relationships (SAR) and improve the antitumour potency of $\mathbf{C 4}$, we introduced new chemical motifs into the scaffold of $\mathbf{C 4}$. These modifications were tested in a preliminary in silico study that suggested an improved interaction with the FAK-FAT domain. The newly designed compounds were synthesised and screened in vitro against three human breast cancer cell lines (MDA-MB-231, BT474 and T47D). Our findings show that compound $\mathbf{5 c}$, which displayed the best anti-proliferative potency amongst all tested analogues, is a novel compound that warrants further investigation as a candidate for breast cancer therapy.

\section{METHODS AND MATERIALS}

\subsection{Lead optimisation and design of novel chloropyramine (C4) analogues}

Based on the previous promising findings with chloropyramine (C4), ${ }^{12,13}$ we sought to improve upon the chemical structure of $\mathbf{C 4}$ to understand better its structure activity relationship (SAR) and to improve its anti-proliferative potency $\left(\mathrm{C}_{4} \mathrm{I} \mathrm{C}_{50}>100 \mu \mathrm{M}\right)$. To investigate the impact of various substituents of $\mathbf{C 4}$ on the antitumour efficacy, a series of novel derivatives were designed and prepared, as detailed in Figure 1, and Schemes 1 and 2. Structural modifications involved replacing the pyridine moiety with other aromatic systems containing a carboxylate moiety to enhance the interaction with Lys 1032 within the FAK-FAT domain, introducing an extended carbon atom moiety (region A, Figure 1) or introducing a carbonyl group into $N, N$-dimethylamino ethyl side chain (region $C$, Figure 1). The $p$-chlorobenzyl group (region B, Figure 1) was retained unmodified in most compounds since it is involved in key interactions via a $\pi-\pi$ stacking with the aromatic side chain of Tyr 925. The salicylate aromatic moiety replacing the pyridine ring was reported to mimic the phosphorylated tyrosine (pTyr). ${ }^{16,17}$ Molecular modeling studies of the newly designed compounds showed improved binding interaction modes within the FAK-FAT pocket compared to the parent C4 compound due to a better orientation effect on the side chain (region C, Figure 1). All the prepared compounds were evaluated against three breast cancer lines i.e. MDA-MB-231 (TNBC), BT474 (ER ${ }^{+}$, $\mathrm{HER}^{+}$) and T47D (ER $\left.{ }^{+}, \mathrm{HER}^{-}\right)$.

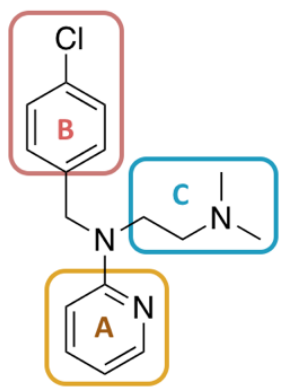

Figure 1. Chemical structure of chloropyramine and regions of proposed structural modification. 


\subsection{Chemical synthesis}

The first series of compounds containing an extended carbon unit in the C4 skeleton (5a-d, region A modification) were synthesised as shown in Scheme 1. The first step involved reductive amination of various substituted aromatic aldehydes (1a-d) with $N, N$-dimethyl-ethylenediamine $\mathbf{2}$ which provided intermediates (3a-d) in good yields. ${ }^{18}$ It is worth mentioning that the reaction conditions of this step were optimized in terms of solvent and temperature choice for the different aldehydes used (see experimental section). These intermediates were alkylated with $p$-chlorobenzyl chloride $\mathbf{4}$ to give the target analogues (5a-d) in good to moderate yield (57-69\%). Interestingly, in the case of 4-((2(dimethylamino)ethylamino)methyl) benzonitrile 3a, a quaternary ammonium side product 6 was obtained alongside the expected product 5a (in 1:3 ratio, respectively). Unexpectedly, in the case of 2cyanobenzaldehyde $\mathbf{7}$, the cyclised isoindolenone derivative 8 was isolated instead of the desired Schiff's base, Scheme 2 (A). A plausible mechanism is suggested where the initial step is the nucleophilic addition of the amine $\mathbf{2}$ to the formyl group of $\mathbf{7}$ affording the adduct $\mathbf{7 a}$, which subsequently cyclises to $\mathbf{7 b}$ by intramolecular nucleophilic addition of the hydroxyl group to the cyano group of $\mathbf{7 a}$, followed by conversion to the isoindolenone derivative $\mathbf{8}$, Scheme $\mathbf{2}$ (B). ${ }^{19}$

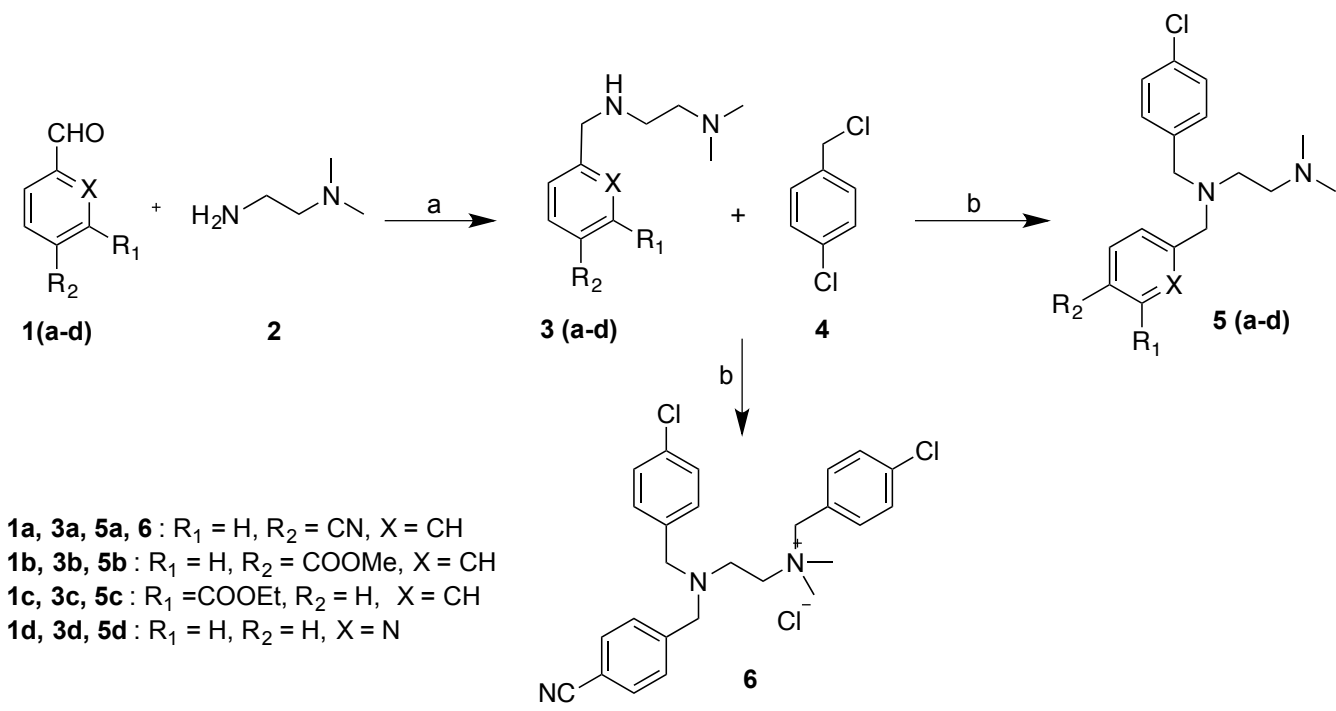

Scheme 1. Synthesis of compounds (5a-d). Reagents and conditions: a) i. 3a and 3d, (MeOH, rt, 4h), 3b (DCE, reflux, 4h), 3c $(\mathrm{AcOH}, 6 \mathrm{~h})$, ii. $\left.\mathrm{NaBH}(\mathrm{OAc})_{3}, \mathrm{rt}, 24 \mathrm{~h}, \mathrm{~b}\right) \mathrm{THF}, \mathrm{rt} .24 \mathrm{~h}$. 


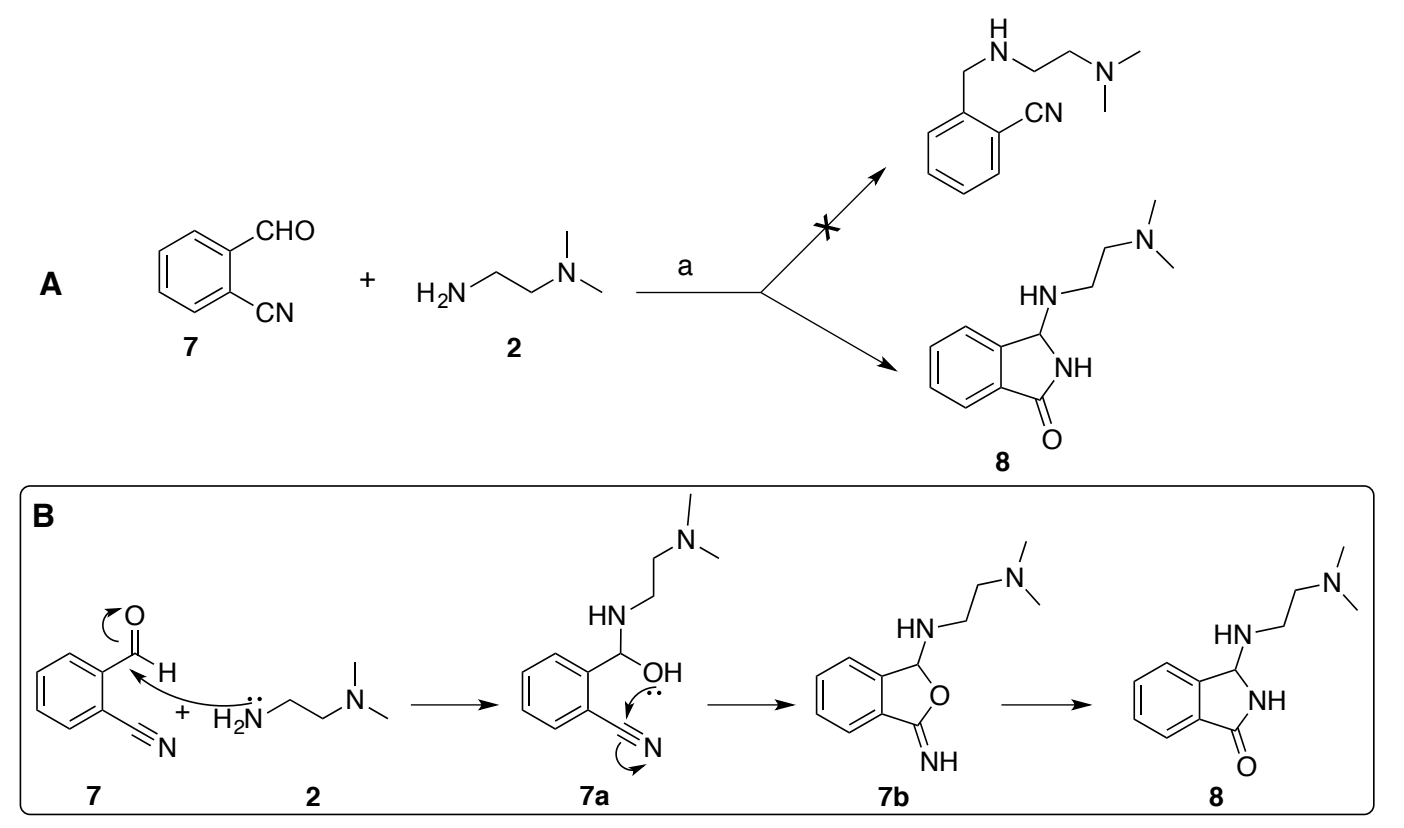

Scheme 2; A) Synthesis of isoindolene derivative 8. Reagents and conditions; a) $\mathrm{MeOH}, \mathrm{rt}, 4 \mathrm{~h}, \mathrm{NaBH}(\mathrm{OAc})_{3}, \mathrm{rt}$, 24h. B) proposed mechanism for the formation of the isoindolenone derivative 8.

The second series of compounds (13a-m), featuring an additional carbonyl group in the $\mathrm{N}, \mathrm{N}$ dimethylamino ethyl side chain moiety of $\mathbf{C 4}$, were prepared according to Scheme 3 (A). The first step involved a reductive amination reaction ${ }^{20}$ between the corresponding aniline derivative (9a-I) and $p$-halobenzaldehyde (10a or $\mathbf{1 0 b})$ to give the key intermediates (11a-I). The final compounds were obtained by reacting $\mathrm{N}, \mathrm{N}$-dimethylaminoacetyl chloride hydrochloride $\mathbf{1 2}$ with intermediates (11a-I) in tetrahydrofuran at room temperature. The reductive amination of 5-amino-2hydroxybenzoic acid with $p$-chlorobenzaldehyde (10a) was not feasible due to the instability of the intermediate product $(11 \mathrm{~m})$. For this reason, we managed to get the corresponding target product $13 \mathrm{~m}$ via the hydrolysis of $13 \mathrm{c}$ using $\mathrm{NaOH}$ solution in a very good yield (92\%), Scheme 3 (A). Interestingly, double reductive amination occurred in the case of compound $\mathbf{9 m}$ to give $\mathbf{1 4}$ which was hydrolysed to 15 using $\mathrm{NaOH}$. The structures of all the synthesised compounds were confirmed using analytical and spectroscopic data (NMR, mass spectrometry), which were in full accordance with their depicted structure. All final compounds were purified and tested as free bases. 
A

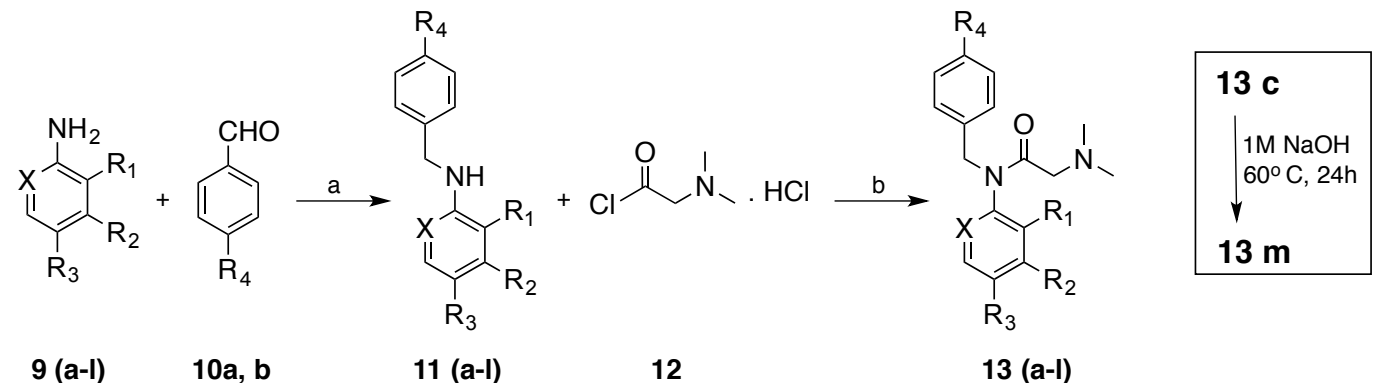

11a, 13a; $\mathrm{R}_{1}=\mathrm{H}, \mathrm{R}_{2}=\mathrm{H}, \mathrm{R}_{3}=\mathrm{H}, \mathrm{R}_{4}=\mathrm{Cl}, \mathrm{X}=\mathrm{N}$

11b, 13b; $R_{1}=H, R_{2}=O H, R_{3}=$ COOMe, $R_{4}=C l, X=C H$

11c, 13c; $\mathrm{R}_{1}=\mathrm{H}, \mathrm{R}_{2}=\mathrm{COOMe}, \mathrm{R}_{3}=\mathrm{OH}, \mathrm{R}_{4}=\mathrm{Cl}, \mathrm{X}=\mathrm{CH}$

11d, 13d; $R_{1}=H, R_{2}=O M e, R_{3}=$ COOMe, $R_{4}=\mathrm{Cl}, X=C H$

11e, 13e; $R_{1}=H, R_{2}=O M e, R_{3}=\mathrm{COOH}, R_{4}=\mathrm{Cl}, X=C H$

11f, 13f; $\mathrm{R}_{1}=\mathrm{H}, \mathrm{R}_{2}=\mathrm{OH}, \mathrm{R}_{3}=\mathrm{COOH}, \mathrm{R}_{4}=\mathrm{Cl}, \mathrm{X}=\mathrm{CH}$

11g, 13g; $\mathrm{R}_{1}=\mathrm{H}, \mathrm{R}_{2}=\mathrm{COOH}, \mathrm{R}_{3}=\mathrm{OMe}, \mathrm{R}_{4}=\mathrm{Cl}, \mathrm{X}=\mathrm{CH}$

11h, 13h; $\mathrm{R}_{1}=\mathrm{H}, \mathrm{R}_{2}=\mathrm{H}, \mathrm{R}_{3}=\mathrm{COOMe}, \mathrm{R}_{4}=\mathrm{Cl}, \mathrm{X}=\mathrm{CH}$

11i, 13i; $\mathrm{R}_{1}=\mathrm{CN}, \mathrm{R}_{2}=\mathrm{H}, \mathrm{R}_{3}=\mathrm{H}, \mathrm{R}_{4}=\mathrm{Cl}, \mathrm{X}=\mathrm{CH}$

11j, 13j; $R_{1}=H, R_{2}=C N, R_{3}=H, R_{4}=C l, X=C H$

11k, 13k; $\mathrm{R}_{1}=\mathrm{H}, \mathrm{R}_{2}=\mathrm{H}, \mathrm{R}_{3}=\mathrm{CN}, \mathrm{R}_{4}=\mathrm{Cl}, \mathrm{X}=\mathrm{CH}$

11I, 13I; $R_{1}=H, R_{2}=C N, R_{3}=H, R_{4}=F, X=C H$

$13 \mathrm{~m} ; \mathrm{R}_{1}=\mathrm{H}, \mathrm{R}_{2}=\mathrm{COOH}, \mathrm{R}_{3}=\mathrm{OH}, \mathrm{R}_{4}=\mathrm{Cl}, \mathrm{X}=\mathrm{CH}$

\section{B}

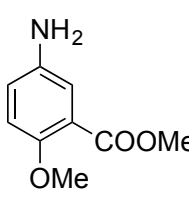

$9 m$<smiles>O=Cc1ccc(Cl)cc1</smiles>

$10 a$<smiles>COC(=O)c1ccc(N(Cc2ccccc2)Cc2ccc(Cl)cc2)cc1</smiles>

14<smiles>COc1ccc(N(Cc2ccc(C)cc2)Cc2ccc(Cl)cc2)cc1C(=O)O</smiles>

15

Scheme 3. A) Synthesis of compounds (13a-m). Reagents and conditions: a) $\left.\mathrm{MeOH}, \mathrm{rt}, 4 \mathrm{~h}, \mathrm{NaBH}(\mathrm{OAc})_{3}, 24 \mathrm{~h}, \mathrm{~b}\right)$ THF, rt. 24h. B) Synthesis of compounds 14 and 15. Reagents and conditions: a) DCE, reflux, 4h, $\mathrm{NaBH}(\mathrm{OAc})_{3}$, r.t. $24 \mathrm{~h}$, b) $\mathrm{NaOH}, \mathrm{MeOH}$, reflux, $4 \mathrm{hr}$.

\section{RESULTS AND DISCUSSION}

\subsection{Cell Viability Assay}

The anti-proliferative activity of all the synthesised compounds 5a-d, 6, 8, 13a-m, 14 and 15 was evaluated as free bases against three human breast cancer cell lines; MDA-MB-231 (TNBC), BT474 $\left(E R^{+}, \mathrm{HER}^{+}\right)$and T47D $\left.\left(E R^{+}, \mathrm{HER}\right)^{-}\right)$. Chloropyramine $\mathrm{C4}$ was used as a control drug. The corresponding cell lines were seeded the day before incubation, then incubated for $72 \mathrm{~h}$ with the different concentrations of compound tested. CellTiter Glo reagent $(25 \mu \mathrm{L})$ was added to the cell plate. The luminescence was recorded after 10 mins on a PerkinElmer Envision instrument. All the twenty-one compounds were tested at 10 concentrations in half log increments up to $100 \mu \mathrm{M}$ in triplicate. The results indicated that three compounds (5c, 6 and 15$)$ have better anti-proliferative activity than chloropyramine $\mathbf{C 4}$, which did not show any anti-proliferative activity up to $100 \mu \mathrm{M}$ concentration across the three $\mathrm{BC}$ cell lines. The most active compound $\mathbf{5 c}$ showed micromolar activity across the three $B C$ cell lines; $\left(M D A-M B-231, I C_{50}=23.5 \mu \mathrm{M}\right),\left(B T 474, I C_{50}=73.2 \mu \mathrm{M}\right)$ and $\left(T 47 D, I C_{50}=31.3\right.$ $\mu \mathrm{M})$, Figure 2A. Interestingly, the quaternary ammonium compound 6 and the double reductive amination product 15, displayed better anti-proliferative activity than C4, Figure $\mathbf{2 B}$ and $\mathbf{C}$, respectively. All the other tested analogues showed no observed improvement in the 
antiproliferative activity over the parent compound C4 (all compounds were tested at maximum concentration of $100 \mu \mathrm{M})$.

A

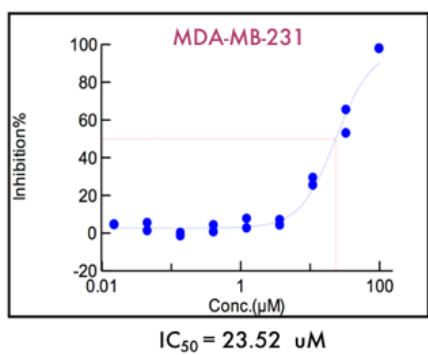

B

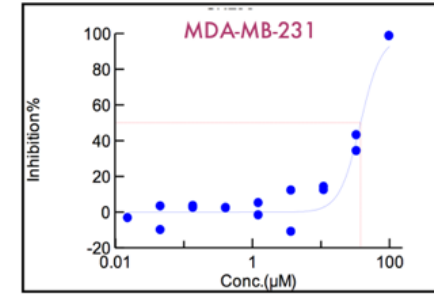

$\mathrm{IC}_{50}=37.45 \mathrm{uM}$

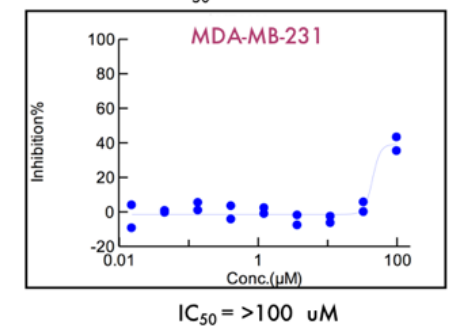

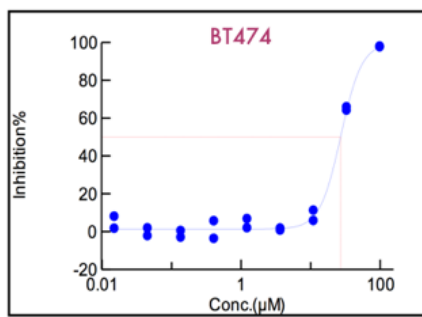

$\mathrm{IC}_{50}=27.15 \mathrm{uM}$

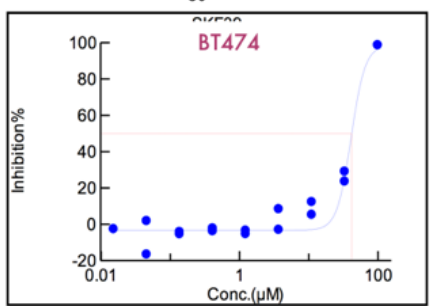

$\mathrm{IC}_{50}=40.87 \mathrm{uM}$

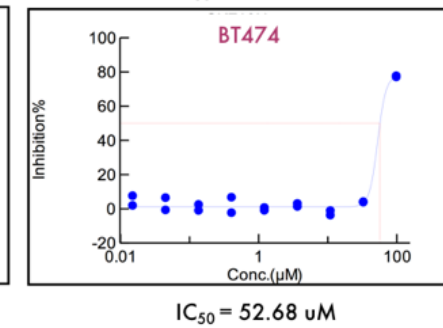

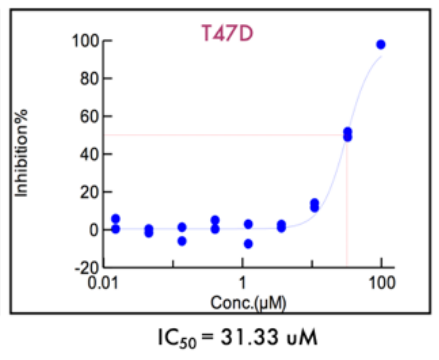

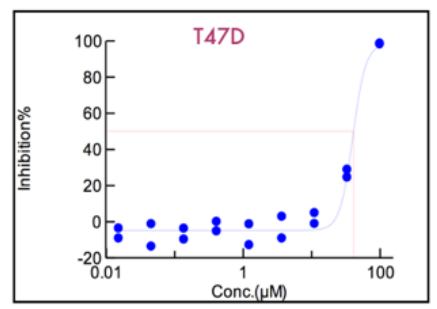

$\mathrm{IC}_{50}=39.81 \mathrm{uM}$

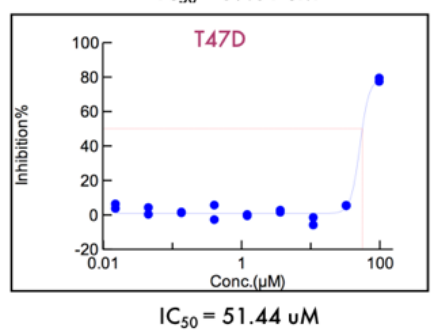

Figure 2. Anti-proliferative activity of compounds $\mathbf{5 C}$ (A), $\mathbf{6}$ (B) and $\mathbf{1 5}$ (C) against three human breast cancer cell lines; MDA-MB-231, BT474 and T47D.

\subsection{Molecular modelling}

In silico docking was performed on all the designed analogues using the downloaded crystal structure of FAK (PDB ID: 1K05). ${ }^{21}$ The chemical structures of the compounds were constructed, rendered and minimised with the MMFF94x force field in MOE. Docking simulations were performed using Glide SP in Maestro (Glide, version 9.5, Schrödinger, LLC, New York, NY. http://www. schrodinger.com). Based on the surface representation of the FAK-FAT domain, there appears to be an improved interaction over the parent $\mathbf{C} \mathbf{4}$ upon the introduction of an extended carbon linker, compounds (5a-d), or introduction of a carbonyl group as in compounds (13a-m) into the general chloropyramine scaffold. Figure $\mathbf{3 A}$ shows the predicted docking mode of the most active compound $\mathbf{5 c}$ compared to that of the parent compound $\mathbf{C 4}$. The computational study showed an improved interaction between the $\mathrm{N}, \mathrm{N}$-dimethylamino ethyl side chain and Asp 1036 of $\mathbf{5 c}$. Compound $\mathbf{5 c}$ sits in a relatively polar cavity surrounded by residues Ser 910, Thr 929, Lys 1032, and Asp 1036. Key interactions of $5 \mathrm{c}$ include two $\mathrm{H}$-bonds between the $\mathrm{N}, \mathrm{N}$-dimethylamino ethyl group and both the side chain carboxylate group of residue Asp 1036 and the backbone carbonyl group of Pro 911. In addition, hydrophobic interactions were observed between the $p$-chlorobenzyl group moiety and the surrounding hydrophobic pocket formed of residues Tyr 925, Val 928, Val 932, and Leu 1035 
(Figure $\mathbf{3 A}$ ). The relatively higher activity of compound $\mathbf{5 c}$ appears to be a result of the better directing effect of the $\mathrm{N}, \mathrm{N}$-dimethylamino side chain conferred by the extra carbon moiety. On the other hand, according to our model, the introduction of the carbonyl group into the $\mathrm{N}, \mathrm{N}$ dimethylamino ethyl moiety of C4 (e.g. 13a) showed an improved effect on orienting the $N, N$ dimethylamino ethyl group into the polar pocket around Asp 1036 as well as directing the other aromatic group to the hydrophobic pocket, Figure 3B. However, none of the carbonyl containing compounds (13a-m) were able to demonstrate any better anti-proliferative activity than C4, probably because of the reduced basicity of the $\mathrm{N}, \mathrm{N}$-dimethylamino moiety upon the introduction of the carbonyl group. Generally, our study of the two modified series of compounds suggests that more substantial structural modifications of $\mathbf{C 4}$ are needed to significantly improve the antiproliferative activity in BC cell lines.
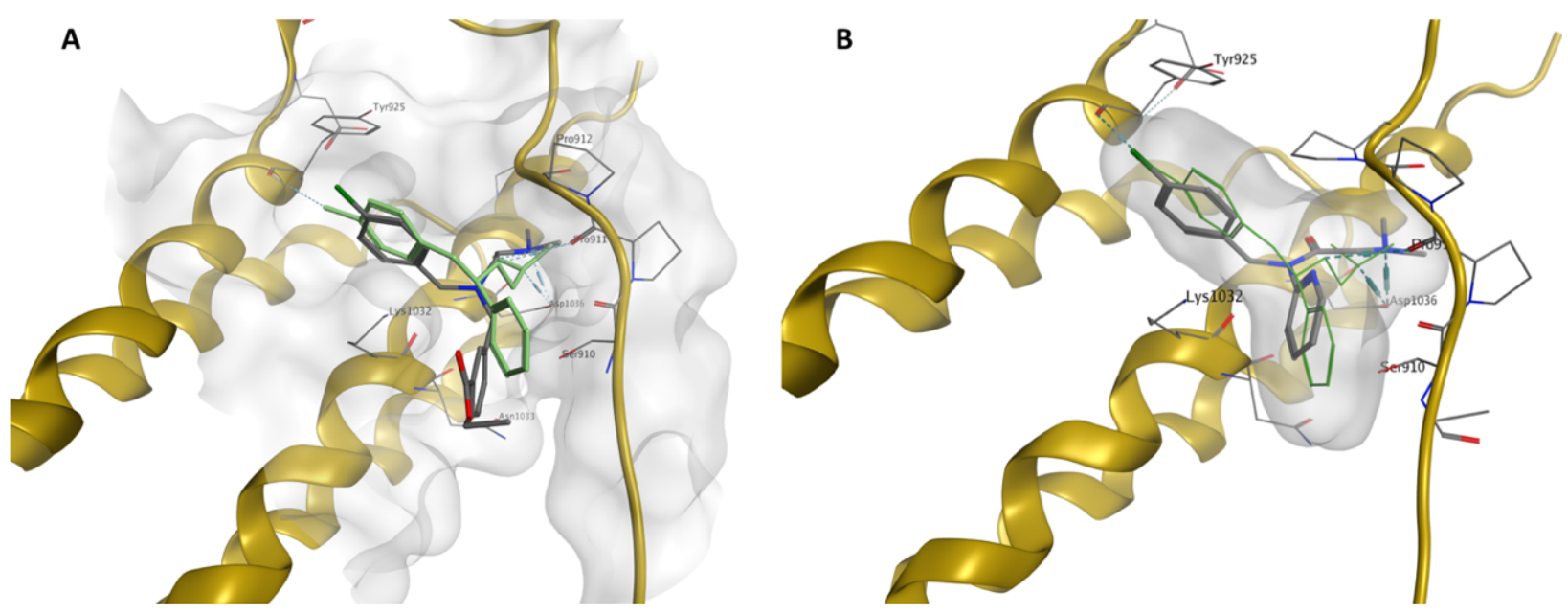

Figure 3. A) Superposition of the predicted binding mode of compound 5c (grey) versus C4 (green) showing hydrogen bond interactions (blue dashed line) with key amino acids; Pro 911 and Asp 1036. B) compound 13a (grey) versus C4 (green); both are inside the pocket of FAK-FAT domain (golden yellow ribbon representation).

\subsection{In vitro aqueous solubility and microsomal metabolic stability}

Subsequently, in vitro pharmacokinetic studies of the most active compound $\mathbf{5 c}$ were performed. The in vitro aqueous solubility was investigated using five concentrations of $5 c$ i.e $(1,3,10,30$ and $100 \mu \mathrm{M}$ ), which were incubated at $37^{\circ} \mathrm{C}$ for $2 \mathrm{hr}$. The estimated precipitation range (lower and upper bound) and a mid-range value were calculated for $\mathbf{5 c}$. Nicardipine and pyrene are included as control compounds. The results show that compound $\mathbf{5 c}$ has a very good aqueous solubility with estimated precipitation range $100 \mu \mathrm{M}$ (lower bound) and $>100 \mu \mathrm{M}$ (upper bound), Table 1. This relatively high aqueous solubility could prove to be a possible solution to the sedation side effect of chloropyramine because of its ability to cross the blood brain barrier (BBB). ${ }^{15}$ The calculated logP values ( $\mathbf{C} 4=3.99, \mathbf{5} \mathbf{c}=4.42)$ are similar but tPSA values $(\mathbf{C} 4=18.84, \mathbf{5} \mathbf{c}=\mathbf{3 2 . 7 8})$ suggest that $\mathbf{5} \mathbf{c}$ is less likely to cross the BBB. 
Furthermore, the metabolic stability of $\mathbf{5 c}$ was tested in human liver microsomes. Compound $\mathbf{5 c}$ was incubated for $45 \mathrm{~min}$ with pooled liver microsomes and the intrinsic clearance (CLint) and half-life $\left(t_{1 / 2}\right)$ values were calculated based on 5 time points. The values of intrinsic clearance $\left(C_{\text {int }}\right)$ as well as half-life values of $\mathbf{5 c}$ compared to control compounds, dextromethorphan and verapamil, are shown in Table 2. Compound $\mathbf{5 c}$ has microsomal half-life $\left(t_{1 / 2}\right)$ of 59.4 minutes, which exceeds that of the registered drug controls dextromethorphan $\left(\mathrm{t}_{1 / 2}=48.2 \mathrm{mins}\right)$ and verapamil $\left(\mathrm{t}_{1 / 2}=8.0 \mathrm{mins}\right)$, and represents a good starting point for optimisation towards a daily administrable product.

\begin{tabular}{|c|c|c|c|}
\hline \multirow{2}{*}{ compound } & \multicolumn{3}{|c|}{ Estimated Precipitation Range $(\boldsymbol{\mu M})$} \\
\cline { 2 - 4 } & Lower bound & Upper bound & Calculated Mid-range \\
\hline 5c & 100 & $>100$ & $>100$ \\
\hline nicardipine & 10 & 30 & 20 \\
\hline pyrene & 3 & 10 & 6.5 \\
\hline
\end{tabular}

Table 1. In vitro aqueous solubility of $\mathbf{5 c}$ versus control compounds (nicardipine and pyrene) in buffered aqueous solution

\begin{tabular}{|c|c|c|}
\hline & $\mathbf{C L}_{\text {int }}(\mathrm{uL} / \mathrm{min} / \mathrm{mg}$ protein) & $\mathbf{t}_{\mathbf{1 / 2}}(\mathrm{min})$ \\
\hline $\mathbf{5 c}$ & 23.3 & 59.4 \\
\hline dextromethorphan & 28.8 & 48.2 \\
\hline verapamil & 173.0 & 8.0 \\
\hline
\end{tabular}

Table 2. In vitro metabolic stability of $\mathbf{5 c}$ versus control compounds (dextromethorphan and verapamil) in human liver microsomes; $\mathrm{CL}_{\text {int }}$ : theoretical unrestricted maximum clearance of unbound drug by an eliminating organ, in absence of blood or plasma protein binding limitations, $\mathrm{t}_{1 / 2}$ is the half-life.

\section{Conclusion}

In this study, two series of novel chloropyramine $\mathbf{C} \mathbf{4}$ analogues were designed and synthesised. The anti-proliferative activity of compounds (5a-d) and (13a-m) was evaluated as free bases in vitro across three human breast cancer cell lines; MDA-MB-231 (TNBC), BT474 (ER $\left.{ }^{+}, \mathrm{HER}^{+}\right)$and T47D $\left(\mathrm{ER}^{+}\right.$, $H_{E R}{ }^{-}$). The results identified compound $\mathbf{5} \mathbf{c}$ as having the most significant anti-proliferative activity, displaying micromolar activity across the three breast cancer cell lines; (MDA-MB-231, $I_{50}=23.5$ $\mu \mathrm{M}),\left(\mathrm{BT} 474, \mathrm{IC}_{50}=73.2 \mu \mathrm{M}\right)$ and $\left(\mathrm{T} 47 \mathrm{D}, \mathrm{IC}_{50}=31.3 \mu \mathrm{M}\right)$. This activity represents a significant improvement to the chloropyramine $\mathbf{C 4}$, which did not display any anti-proliferative activity up to $100 \mu \mathrm{M}$. Further pharmacokinetic study showed that $5 \mathrm{c}$ performed well in both the aqueous solubility and microsomal metabolic stability analysis. Considering the above findings, it could be concluded that $\mathbf{5 c}$ represents a promising basis for further development of breast cancer therapeutics targeting the FAK scaffolding domain. 


\section{Acknowledgement}

We thank the Welsh Government, Life Sciences Research Network Wales scheme for postdoctoral support to SK. Anti-proliferative assays were run as an outsourced service through WuxiApptech AppTec Co., Ltd. All in vitro biological evaluations were performed by Cyprotex, and the detailed protocols can be found at http://www.cyprotex.com. We also thank the EPSRC National Mass Spectrometry centre (Swansea, U.K.) for provision of accurate mass spectrometry. We thank the analytical services unit, school of Chemistry, Cardiff University for provision of accurate mass spectrometry. We thank Miss. Emma Jones for the technical support.

\section{Supplementary data}

Details of experimental procedures and spectroscopic characterisations data of the compounds $\mathbf{5 a -}$

d, 6, 8, 13a-m, 14 and 15 associated with this article can be found in the online version at

\section{References}

1. Breast cancer: prevention and control. World Health Organization. http:// www.who.int/cancer/detection/breastcancer/en/index1.html.

2. F. J. Sulzmaier, C. Jean, D. D. Schlaepfer, Nature, 2014, 14, 598.

3. V.G. Brunton, M. Frame Curr. Opin. Pharmacol, 2008, 8, 427.

4. V. Gabarra-Niecko, M. D. Schaller, J. M. Dunty, Cancer Metastasis Rev, 2003, 22, 359.

5. Y. Y. Chen, Z. X. Wang, P. A. Chang, J. J. Li, F. Pan, L. Yang, Z. H. Bian, L. Zou, J. M. He, H. J. Liang. Cancer Sci, 2009, 100, 1708.

6. W.G. Cance, E. Kurenova, T. Marlowe, V. Golubovskaya, Sci. Signal. 2013, 6, 10.

7. G.W. McLean, N.O. Carragher, E. Avizienyte, J. Evans, V.G. Brunton, M.C. Frame, Nat. Rev. Cancer, 2005, 5, 505.

8. M. Keegan, J.E. Ring, V.N. Kolev, I.M. Shapiro, M.V. Padval, Q. Xu, J.A. Pachter, Ann Oncol, 2014, 25,4 , iv555.

9. J. Halder, Y. G. Lin, W. M. Merritt, W. A. Spannuth, A. M. Nick, T. Honda, A. A. Kamat, L.Y. Han, T. J. Kim, C. Lu, A. M. Tari, W. Bornmann, A. Fernandez, G. Lopez-Berestein, A. K. Sood, Cancer Res, 2007, 67, 10976.

10. J. B. Stokes, S. J. Adair, J. Slack-Davis, D. M. Walters, R. W. Tilghman, E. D. Hershey, B Lowrey, K. S. Thomas, A. H. Bouton, R. F. Hwang, E. B. Stelow, J. T. Parsons, T. W. Bauer, Mol Cancer Ther, 2011, 10, 2135.

11. A. Schultze, W. Fiedler, Anticancer Agents Med Chem, 2011, 11, 593.

12. E. V. Kurenova, D. L. Hunt, D. He, A. T. Magis, D. A. Ostrov, W. G. Cance, J Med Chem, 2009, 52, 4716.

13. P. N. Gogate, M. Ethirajan, E. V. Kurenova, A. T. Magisc, R. K. Pandeya, W. G. Cance, Eur J Med Chem, 2014, 80, 154.

14. J. Wilton, E. Kurenova, L. Pitzonka, A. Gaudy, L. Curtin, S. Sexton, W. Cance, G. Fetterly, Eur J Drug Metab Pharmacokinet, 2016, 41, 55.

15. J. T. Burgess, E. Bolderson, J. M. Saunus, S. Zhang, L. E. Reid, A. M. McNicol, S. R. Lakhani, K. Cuff, K. Richard, D. J. Richard, K. J. O'Byrne, Oncotarget, 2016, 8;7, 45, 72807.

16. B.D.G. Page, D. C. Croucher, Z. L. Hua, S. Haftchenary, V. H. Jimenez-Zepeda, J. Atkinson, P. A. Spagnuolo, Y. L. Wong, R. Colaguori, A. M. Lewis, A. D. Schimmer, S. Trudel, P. T. Gunning, J Med Chem, 2013, 56, 7190.

17. P. Morlacchi, F. M. Robertson, J. Klostergaard, J. S. McMurray, Future Med Chem, 2014; 6(17), 1909.

18. J. Lasri, C. M. Fátima, G. da Silva, M. N. Kopylovich, B. G. Mukhopadhyay, A. J. L. Pombeiro, Eur J 
Inorg Chem, 2009, 5541.

19. R. Sato, T. Senzaki, T. Goto, M. Saito, Chemistry letters, 1984, 1599.

20. P. Wiklund, J. Bergman, Org Biomol Chem, 2003, 1, 367.

21. S.T. Arold, M.K. Hoellerer, M.E. Noble, Structure, 2002, 10, 319. 


\title{
Supporting Information
}

\section{The discovery of new and more potent chloropyramine (C4) analogues for the potential treatment of invasive breast cancer}

\author{
Sahar Kandil ${ }^{*}$, Filippo Prencipe, Samuel Jones, Stephen Hiscox and Andrew D. Westwell \\ School of Pharmacy \& Pharmaceutical Sciences, Cardiff University, King Edward VII Avenue, Cardiff, CF10 3NB, \\ Wales, United Kingdom.
}

Keywords: Focal adhesion kinase (FAK), metastatic breast cancer (BC), chloropyramine (C4), lead optimisation, reductive amination.

All chemicals were purchased from Sigma-Aldrich or Alfa Aesar and were used without further purification. Thin Layer Chromatography (TLC): pre-coated aluminium backed plates $\left(60 \mathrm{~F}_{254}, 0.2 \mathrm{~mm}\right.$ thickness, Merck) were visualized under both short and long wave UV light (254 and $366 \mathrm{~nm}$ ). Flash column chromatography was carried out using silica gel supplied by Fisher (60A, 35-70 $\mu \mathrm{m}) .{ }^{1} \mathrm{H}$ NMR $(500 \mathrm{MHz}),{ }^{13} \mathrm{C}$ NMR $(125 \mathrm{MHz})$ and ${ }^{19} \mathrm{~F}$ NMR $(470 \mathrm{MHz})$ spectra were recorded on a Bruker Avance $500 \mathrm{MHz}$ spectrometer at $25^{\circ} \mathrm{C}$. Chemical shifts $(\delta)$ are expressed in parts per million (ppm) and coupling constants $(\mathrm{J})$ are given in hertz $(\mathrm{Hz})$. The following abbreviations are used in the assignment of NMR signals: s (singlet), bs (broad singlet); $d$ (doublet), $t$ (triplet), q (quartet), qn (quintet), $m$ (multiplet), dd (doublet of doublet), dt (doublet of triplet), td (triple doublet); dq (double quartet), $m$ (multiplet), dm (double multiplet). Mass spectrometry was performed on a Bruker Daltonics microTof-LC system or as a service through the EPSRC National Mass Spectrometry centre (Swansea, UK).

4-((2-(Dimethylamino)ethylamino)methyl)benzonitrile (3a) yield $94 \%$. To a solution of 4cyanobenzaldehyde (1a; $1.5 \mathrm{mmol}$ ) in $\mathrm{MeOH}(25 \mathrm{~mL}$ ) was added dropwise 2-dimethylaminoethyl amine $(2 ; 1.8 \mathrm{mmol})$. The solution was stirred at room temperature for $4 \mathrm{~h}$, sodium borohydride $(0.75 \mathrm{mmol}$ ) was added, and the mixture was stirred at room temperature for $24 \mathrm{~h}$. The progress of the reaction was monitored by TLC. The solution was then dried in vacuo, and the crude residue was purified by column chromatography eluting with $\mathrm{CH}_{2} \mathrm{Cl}_{2} .{ }^{1} \mathrm{H} \mathrm{NMR}$ (MeOD) $\delta 7.69(\mathrm{~d}, \mathrm{~J}=8 \mathrm{~Hz}, 2 \mathrm{H}, \mathrm{ArH}$ ), $7.55(\mathrm{~d}, \mathrm{~J}=7.5 \mathrm{~Hz}, 2 \mathrm{H}, \operatorname{ArH}), 4.84$ (bs, $1 \mathrm{H}, \mathrm{NH}), 3.86\left(\mathrm{~s}, 2 \mathrm{H}, \mathrm{CH}_{2}\right), 2.71$ (t, J=5.5 Hz, 2H, CH $\left.)_{2}\right), 2.50$ (t, J=6 $\left.\mathrm{Hz}, 2 \mathrm{H}, \mathrm{CH}_{2}\right), 2.25\left(\mathrm{~s}, 6 \mathrm{H}, 2 \times \mathrm{CH}_{3}\right),{ }^{13} \mathrm{C}$ NMR (MeOD) $\delta 145.60(\mathrm{ArC}), 131.99(\mathrm{ArCH}), 128.95(\mathrm{ArCH})$, $118.50(\operatorname{ArCN}), 110.49(\operatorname{ArC}), 58.26\left(\mathrm{CH}_{2}\right), 52.65\left(\mathrm{CH}_{2}\right), 45.89\left(\mathrm{CH}_{2}\right), 44.40\left(2 \mathrm{CH}_{3}\right) . \mathrm{MS}$ [ESI, m/z]: 204.1 $[\mathrm{M}+\mathrm{H}]$.

\footnotetext{
* Corresponding author E-mail address: Kandils1@cardiff.ac.uk (S. Kandil)
} 
Methyl 4-(((2-(dimethylamino)ethyl)amino)methyl)benzoate (3b) yield $92 \%$. To a solution of methyl 4-formylbenzoate (1); $1.5 \mathrm{mmol})$ in DCE $(25 \mathrm{~mL})$ was added dropwise 2dimethylaminoethylamine $(2 ; 1.8 \mathrm{mmol})$. The solution was heated at reflux for $4 \mathrm{~h}$, sodium triacetoxyborohydride $(2.4 \mathrm{mmol})$ was then added to the mixture which was stirred at room temperature for $24 \mathrm{~h}$. The progress of the reaction was monitored by TLC. The solution was then dried in vacuo, and the crude residue was purified by column chromatography eluting with $\mathrm{CH}_{2} \mathrm{Cl}_{2} / \mathrm{CH}_{3} \mathrm{OH}$; 90:10. ${ }^{1} \mathrm{H}$ NMR (MeOD) 7.99 (d, J=8.5 Hz, 2H, ArH), 7.48 (d, J=8 Hz, 2H, ArH), 3.91 (s, $\left.3 \mathrm{H}, \mathrm{CH}_{3}\right), 3.85\left(\mathrm{~s}, 2 \mathrm{H}, \mathrm{CH}_{2}\right), 2.72\left(\mathrm{t}, \mathrm{J}=7 \mathrm{~Hz}, 2 \mathrm{H}, \mathrm{CH}_{2}\right), 2.51\left(\mathrm{t}, \mathrm{J}=7 \mathrm{~Hz}, 2 \mathrm{H}, \mathrm{CH}_{2}\right), 2.25\left(\mathrm{~s}, 6 \mathrm{H}, 2 \times \mathrm{CH}_{3}\right) .{ }^{13} \mathrm{C}$ NMR (MeOD) $\delta 166.91(C=0), 144.95(\operatorname{ArC}), 129.34(\mathrm{ArCH}), 128.86(\operatorname{ArC}), 128.18(\mathrm{ArCH}), 57.99\left(\mathrm{CH}_{2}\right)$, $52.62\left(\mathrm{CH}_{2}\right), 51.23\left(\mathrm{CH}_{3}\right), 45.55\left(\mathrm{CH}_{2}\right), 44.17\left(2 \mathrm{CH}_{3}\right) . \mathrm{MS}[\mathrm{ESI}, \mathrm{m} / \mathrm{z}]: 237.1[\mathrm{M}+\mathrm{H}]$.

Ethyl 4-(((2-(dimethylamino)ethyl)amino)methyl)benzoate (3c) yield $92 \%$. To a solution of ethyl 3formylbenzoate (1c; $1.5 \mathrm{mmol}$ ) in $\mathrm{AcOH}(25 \mathrm{~mL}$ ) was added dropwise 2-dimethylaminoethylamine (2; $1.8 \mathrm{mmol}$ ). The solution was stirred at room temperature for $6 \mathrm{~h}$, then sodium triacetoxyborohydride $(2.4 \mathrm{mmol}$ ) was added to the mixture which was stirred at room temperature for $24 \mathrm{~h}$. The progress of the reaction was monitored by TLC. The solution was then dried in vacuo, and the crude residue was purified by column chromatography eluting with $\mathrm{CH}_{2} \mathrm{Cl}_{2} / \mathrm{CH}_{3} \mathrm{OH}$ 90:10. ${ }^{1} \mathrm{H} \mathrm{NMR}\left(\mathrm{CDCl}_{3}\right) 7.99$ (s, $1 \mathrm{H}, \operatorname{ArH}), 7.92(\mathrm{~d}, \mathrm{~J}=8 \mathrm{~Hz}, 1 \mathrm{H}, \operatorname{ArH}), 7.54(\mathrm{~d}, J=8 \mathrm{~Hz}, 1 \mathrm{H}, \operatorname{ArH}), 7.38(\mathrm{t}, J=7.5 \mathrm{~Hz}, 1 \mathrm{H}, \operatorname{ArH}), 4.37$ (q, J=7 $\left.\mathrm{Hz}, 2 \mathrm{H}, \mathrm{CH}_{2}\right), 3.86\left(\mathrm{~s}, 2 \mathrm{H}, \mathrm{CH}_{2}\right), 2.72\left(\mathrm{t}, \mathrm{J}=6 \mathrm{~Hz}, 2 \mathrm{H}, \mathrm{CH}_{2}\right), 2.49\left(\mathrm{t}, \mathrm{J}=6 \mathrm{~Hz}, 2 \mathrm{H}, \mathrm{CH}_{2}\right), 2.24\left(\mathrm{~s}, 6 \mathrm{H}, 2 \mathrm{CH}_{3}\right)$. ${ }^{13} \mathrm{C} \mathrm{NMR}\left(\mathrm{CDCl}_{3}\right) \delta 166.60(\mathrm{C}=\mathrm{O}), 140.36(\mathrm{ArC}), 132.69(\mathrm{ArCH}), 130.58(\mathrm{ArC}), 129.22(\mathrm{ArCH}), 128.42$ $(\mathrm{ArCH}), 128.23(\mathrm{ArCH}), 60.91\left(\mathrm{CH}_{2}\right), 58.56\left(\mathrm{CH}_{2}\right), 53.42\left(\mathrm{CH}_{2}\right), 46.09\left(\mathrm{CH}_{2}\right), 45.13\left(2 \times \mathrm{CH}_{3}\right), 14.47\left(\mathrm{CH}_{3}\right)$. MS [ESI, m/z]: calcd for $\mathrm{C}_{14} \mathrm{H}_{22} \mathrm{~N}_{2} \mathrm{O}_{2}[\mathrm{M}+\mathrm{H}], 251.1754$; found, 251.1754 [M+H], $273.1574\left[\mathrm{M}+\mathrm{Na}^{+}\right]$.

$\mathbf{N}^{\prime}, \mathbf{N}^{\prime}$-dimethyl-N2-(pyridine-2-ylmethyl)ethane-1,2-diamine (3d), yield 94\%. To a solution of pyridine-2-carboxaldehyde (1d; $1.5 \mathrm{mmol})$ in $\mathrm{MeOH}(25 \mathrm{~mL})$ was added dropwise 2dimethylaminoethyl amine $(2 ; 1.8 \mathrm{mmol})$. The solution was stirred at room temperature for $4 \mathrm{~h}$, then sodium triacetoxyborohydride $(2.4 \mathrm{mmol})$ was added to the mixture which was stirred at room temperature for $24 \mathrm{~h}$. The progress of the reaction was monitored by TLC. The solution was then dried in vacuo, and the crude residue was purified by column chromatography eluting with $\mathrm{CH}_{2} \mathrm{Cl}_{2} / \mathrm{CH}_{3} \mathrm{OH}$ 90:10. ${ }^{1} \mathrm{H}-\mathrm{NMR}\left(\mathrm{CDCl}_{3}\right) \delta 8.55(\mathrm{~s}, 1 \mathrm{H}, \mathrm{ArH}), 7.69(\mathrm{t}, J=8 \mathrm{~Hz}, 1 \mathrm{H}, \mathrm{ArH}), 7.36(\mathrm{~d}, J=8 \mathrm{~Hz}$,

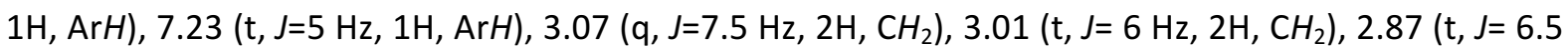
$\left.\mathrm{Hz}, 2 \mathrm{H}, \mathrm{CH}_{2}\right), 2.48\left(\mathrm{~s}, 6 \mathrm{H}, 2 \times \mathrm{CH}_{3}\right), 1.99(\mathrm{~s}, 1 \mathrm{H}, \mathrm{NH}) . \mathrm{MS}[\mathrm{ESI}, \mathrm{m} / \mathrm{z}]: 271.1[\mathrm{M}+\mathrm{H}]$.

4-(((4-Chlorobenzyl)(2-(dimethylamino)ethyl)amino)methyl)benzonitrile (5a) yield 65 \%. A mixture of compound 3a (1.22 mmol), $\mathrm{K}_{2} \mathrm{CO}_{3}(3.65 \mathrm{mmol})$, and 1-chloro-4-(chloromethyl)benzene 4a (2.31 $\mathrm{mmol}$ ) in anhydrous tetrahydrofuran $(50 \mathrm{~mL}$ ) was stirred at room temperature for $24 \mathrm{~h}$. the reaction mixture was evaporated in vacuo then extracted with ethyl acetate $(2 \times 50 \mathrm{~mL})$. The organic layer 
was dried then evaporated and purified by column chromatography eluting with $\mathrm{CH}_{2} \mathrm{Cl}_{2} / \mathrm{CH}_{3} \mathrm{OH}$ 90:10. ${ }^{1} \mathrm{H}$ NMR (MeOD) $\delta 7.69$ (d, J=8.5 Hz, 2H, ArH), 7.67 (d, J=8.5 Hz, 2H, ArH), 7.62 (d, J=8.5 Hz, 2H, $\operatorname{ArH}), 7.52(\mathrm{~d}, \mathrm{~J}=8.5 \mathrm{~Hz}, 2 \mathrm{H}, \operatorname{ArH}), 4.76\left(\mathrm{~s}, 2 \mathrm{H}, \mathrm{CH}_{2}\right), 3.40\left(\mathrm{~s}, 2 \mathrm{H}, \mathrm{CH}_{2}\right), 3.62\left(\mathrm{t}, J=6.5 \mathrm{~Hz}, 2 \mathrm{H}, \mathrm{CH}_{2}\right), 3.22$ $\left(\mathrm{t}, \mathrm{J}=6.5 \mathrm{~Hz}, 2 \mathrm{H}, \mathrm{CH}_{2}\right), 3.20\left(\mathrm{~s}, 6 \mathrm{H}, 2 \times \mathrm{CH}_{3}\right),{ }^{13} \mathrm{C}$ NMR (MeOD) $\delta 145.91(\operatorname{ArC}), 136.67(\operatorname{ArC}), 134.46$ ( $\mathrm{ArCH}), 132.32(\mathrm{ArCH}), 128.78(\mathrm{ArCH}), 127.27(\mathrm{ArCH}), 126.41(\operatorname{ArC}), 118.76(\operatorname{ArC}), 110.27(\operatorname{ArC}), 67.59$ $\left(\mathrm{CH}_{2}\right), 63.26\left(\mathrm{CH}_{2}\right), 52.51\left(\mathrm{CH}_{2}\right), 49.60\left(2 \times \mathrm{CH}_{3}\right), 42.60\left(\mathrm{CH}_{2}\right)$. MS [ESI, m/z]: calcd for $\mathrm{C}_{19} \mathrm{H}_{22} \mathrm{ClN}_{3}[\mathrm{M}+\mathrm{H}]$, 328.1581; found, 328.1566 .

Methyl 4-(((4-chlorobenzyl)(2-(dimethylamino)ethyl)amino)methyl) benzoate (5b) yield $57 \%$. A mixture of compound $\mathbf{3 b}(1.22 \mathrm{mmol}), \mathrm{K}_{2} \mathrm{CO}_{3}(3.65 \mathrm{mmol})$, and 1-chloro-4 (chloromethyl)benzene 4a (2.31 mmol) in anhydrous THF $(50 \mathrm{~mL})$ was stirred at room temperature for $24 \mathrm{~h}$. the reaction mixture was evaporated in vacuo then extracted with ethyl acetate $(2 \times 50 \mathrm{~mL})$. The organic layer was dried then evaporated and purified by column chromatography eluting with $\mathrm{CH}_{2} \mathrm{Cl}_{2} / \mathrm{CH}_{3} \mathrm{OH}$ 90:10. ${ }^{1} \mathrm{H}$ NMR $\left(\mathrm{CDCl}_{3}\right) \delta 8.01(\mathrm{~d}, J=8.5 \mathrm{~Hz}, 2 \mathrm{H}, \operatorname{ArH}), 7.45(\mathrm{~d}, J=8.5 \mathrm{~Hz}, 2 \mathrm{H}, \operatorname{ArH}), 7.31(\mathrm{~s}, 2 \mathrm{H}, \operatorname{ArH}), 7.30$ (d, J=9 Hz, 2H, ArH), $3.93\left(\mathrm{~s}, 3 \mathrm{H}, \mathrm{CH}_{3}\right), 3.66\left(\mathrm{~s}, 2 \mathrm{H}, \mathrm{CH}_{2}\right), 3.59\left(\mathrm{~s}, 2 \mathrm{H}, \mathrm{CH}_{2}\right), 2.59\left(\mathrm{~m}, 2 \mathrm{H}, \mathrm{CH}_{2}\right), 2.48(\mathrm{~m}$, $\left.2 \mathrm{H}, \mathrm{CH}_{2}\right), 2.20\left(\mathrm{~s}, 6 \mathrm{H}, 2 \times \mathrm{CH}_{3}\right) .{ }^{13} \mathrm{C}$ NMR $\left(\mathrm{CDCl}_{3}\right) \delta 167.07(\mathrm{C}=\mathrm{O}), 145.04(\operatorname{ArC}), 137.83(\mathrm{ArC}), 132.69$ $(\operatorname{ArC}), 130.03(\mathrm{ArCH}), 129.62(\mathrm{ArCH}), 128.95(\mathrm{ArC}), 128.58(\mathrm{ArCH}), 128.42(\mathrm{ArCH}), 58.52\left(\mathrm{CH}_{2}\right), 58.20$ $\left(\mathrm{CH}_{2}\right), 57.48\left(\mathrm{CH}_{2}\right), 52.05\left(\mathrm{CH}_{3}\right), 51.53\left(\mathrm{CH}_{2}\right), 45.63\left(2 \times \mathrm{CH}_{3}\right)$. MS [ESI, m/z]: calcd for $\mathrm{C}_{20} \mathrm{H}_{25} \mathrm{ClN}_{2} \mathrm{O}_{2}$ $[\mathrm{M}+\mathrm{H}], 361.1677$; found, $361.1679[\mathrm{M}+\mathrm{H}], 383.1497[\mathrm{M}+\mathrm{Na}]$.

Ethyl 3-(((4-chlorobenzyl)(2-(dimethylamino)ethyl)amino)methyl) benzoate (5c) yield $61 \%$ A mixture of compound $3 \mathrm{c}(1.22 \mathrm{mmol}), \mathrm{K}_{2} \mathrm{CO}_{3}(3.65 \mathrm{mmol})$, and 1-chloro-4 (chloromethyl)benzene $4 \mathrm{a}$ $(2.31 \mathrm{mmol})$ in anhydrous tetrahydrofuran $(50 \mathrm{~mL})$ was stirred at room temperature for $24 \mathrm{~h}$. the reaction mixture was evaporated in vacuo then extracted with ethyl acetate $(2 \times 50 \mathrm{~mL})$. The organic layer was dried then evaporated and purified by column chromatography eluting with $\mathrm{CH}_{2} \mathrm{Cl}_{2} / \mathrm{CH}_{3} \mathrm{OH}$ 90:10. ${ }^{1} \mathrm{H}$ NMR $\left(\mathrm{CDCl}_{3}\right) \delta 8.03-8.01(\mathrm{~m}, 1 \mathrm{H}, \mathrm{ArH}), 7.94(\mathrm{dd}, J=8,1.5 \mathrm{~Hz}, 1 \mathrm{H}, \operatorname{ArH}), 7.58$ (ddd, J=7.5, 2, $1.5 \mathrm{~Hz}, 1 \mathrm{H}, \operatorname{Ar} H), 7.41(\mathrm{~d}, J=8 \mathrm{~Hz}, 1 \mathrm{H}, \operatorname{ArH}), 7.32-7.28(\mathrm{~m}, 4 \mathrm{H}, \operatorname{ArH}), 4.41\left(\mathrm{q}, J=7 \mathrm{~Hz}, 2 \mathrm{H}, \mathrm{CH}_{2}\right), 3.66(\mathrm{~s}$, $\left.2 \mathrm{H}, \mathrm{CH}_{2}\right), 3.59\left(\mathrm{~s}, 2 \mathrm{H}, \mathrm{CH}_{2}\right), 2.63\left(\mathrm{t}, \mathrm{J}=8 \mathrm{~Hz}, 2 \mathrm{H}, \mathrm{CH}_{2}\right), 2.51\left(\mathrm{t}, \mathrm{J}=8 \mathrm{~Hz}, 2 \mathrm{H}, \mathrm{CH}_{2}\right), 2.22\left(\mathrm{~s}, 6 \mathrm{H}, 2 \mathrm{CH}_{3}\right), 1.43$ $\left(\mathrm{t}, J=7.5 \mathrm{~Hz}, 3 \mathrm{H}, \mathrm{CH}_{3}\right) .{ }^{13} \mathrm{C}$ NMR $\left(\mathrm{CDCl}_{3}\right) \delta 166.70(\mathrm{C}=\mathrm{O}), 139.73(\operatorname{ArC}), 137.88(\operatorname{ArC}), 133.21(\operatorname{ArCH})$, $132.67(\mathrm{ArC}), 130.50(\mathrm{ArC}), 130.07(\mathrm{ArCH}), 129.78(\mathrm{ArCH}), 128.42(\mathrm{ArCH}), 128.35(\mathrm{ArCH}), 128.30$ $(\operatorname{ArCH}), 60.98\left(\mathrm{CH}_{2}\right), 58.39\left(\mathrm{CH}_{2}\right), 58.08\left(\mathrm{CH}_{2}\right), 57.43\left(\mathrm{CH}_{2}\right), 51.31\left(\mathrm{CH}_{2}\right), 45.59\left(2 \mathrm{CH}_{3}\right), 14.36\left(\mathrm{CH}_{3}\right) . \mathrm{MS}$ [ESI, m/z]: calcd for $\mathrm{C}_{21} \mathrm{H}_{27} \mathrm{CIN}_{2} \mathrm{O}_{2}[\mathrm{M}+\mathrm{H}], 375.1834$; found, $375.1838[\mathrm{M}+\mathrm{H}], 397.1650[\mathrm{M}+\mathrm{Na}]$.

$\mathbf{N}^{1}$-(4-chlorobenzyl)-N $\mathbf{N}^{2}, \mathbf{N}^{2}$-dimethyl- $\mathbf{N}^{1}$-(pyridin-2-ylmethyl)ethane-1,2-diamine (5d) yield $69 \%$. A mixture of compound $3 \mathrm{~d}(1.22 \mathrm{mmol}), \mathrm{K}_{2} \mathrm{CO}_{3}(3.65 \mathrm{mmol})$, and 1-chloro-4-(chloromethyl)benzene $4 \mathrm{a}$ $(2.31 \mathrm{mmol})$ in anhydrous tetrahydrofuran $(50 \mathrm{~mL})$ was stirred at room temperature for $24 \mathrm{~h}$. the reaction mixture was evaporated in vacuo then extracted with ethyl acetate $(2 \times 50 \mathrm{~mL})$. The organic 
layer was dried then evaporated and purified by column chromatography eluting with $\mathrm{CH}_{2} \mathrm{Cl}_{2} / \mathrm{CH}_{3} \mathrm{OH}$ 90:10. ${ }^{1} \mathrm{H}$ NMR $\left(\mathrm{CDCl}_{3}\right) \delta 8.40$ (ddd, $\left.J=5,2,1 \mathrm{~Hz}, 1 \mathrm{H}, \mathrm{ArH}\right), 7.67$ (td, $\left.J=8,2 \mathrm{~Hz}, 1 \mathrm{H}, \mathrm{ArH}\right), 7.67$ (d, J= 7.5 $\mathrm{Hz}, 1 \mathrm{H}, \operatorname{ArH}$ ), 7.33 (d, J=8.5 Hz, 2H, ArH), 7.29 (d, J=9 Hz, 2H, ArH), 7.16 (ddd, J= 7.5, 5,1 Hz, 1H, $\operatorname{ArH}$ ), $3.79\left(\mathrm{~s}, 2 \mathrm{H}, \mathrm{CH}_{2}\right.$ ), $3.65\left(\mathrm{~s}, 2 \mathrm{H}, \mathrm{CH}_{2}\right), 2.63\left(\mathrm{dd}, J=9,7.5 \mathrm{~Hz}, 2 \mathrm{H}, \mathrm{CH}_{2}\right.$ ), 2.47 (dd, J= 9, $7.5 \mathrm{~Hz}, 2 \mathrm{H}$, $\left.\mathrm{CH}_{2}\right), 2.18\left(\mathrm{~s}, 6 \mathrm{H}, 2 \mathrm{CH}_{3}\right) .{ }^{13} \mathrm{C} \mathrm{NMR}\left(\mathrm{CDCl}_{3}\right) \delta 159.91(\mathrm{ArC}), 148.95(\mathrm{ArCH}), 137.90(\mathrm{ArC}), 136.44(\mathrm{ArCH})$, $132.58(\mathrm{ArC}), 130.10(\mathrm{ArCH}), 128.37(\mathrm{ArCH}), 122.82(\mathrm{ArCH}), 121.97(\mathrm{ArCH}), 60.50\left(\mathrm{CH}_{2}\right), 58.38\left(\mathrm{CH}_{2}\right)$, $57.53\left(\mathrm{CH}_{2}\right), 51.93\left(\mathrm{CH}_{2}\right), 45.83\left(\mathrm{CH}_{3}\right) .[\mathrm{ESI}, \mathrm{m} / \mathrm{z}]: 304.1576[\mathrm{M}+\mathrm{H}], 326.1396[\mathrm{M}+\mathrm{Na}]$. calcd for $\mathrm{C}_{17} \mathrm{H}_{22} \mathrm{ClN}_{3}[\mathrm{M}+\mathrm{H}], 304.1575$; found, 304.1576 .

\section{$\mathrm{N}$-(4-((4-chlorobenzyl)(2-(dimethylamino)ethyl)amino)methyl)benzylidyne)-1-(4-chlorophenyl)}

methanaminium (6) yield $21 \% .{ }^{1} \mathrm{H}$ NMR $\left(\mathrm{CDCl}_{3}\right) \delta 7.63(\mathrm{~d}, J=8.5 \mathrm{~Hz}, 2 \mathrm{H}, \mathrm{ArH}), 7.48(\mathrm{~d}, J=8 \mathrm{~Hz}, 2 \mathrm{H}$, $\operatorname{ArH}$ ), 7.45 (d, J=8.5 Hz, 2H, ArH), 7.35 (d, J=8.5 Hz, 2H, ArH), 7.30 (d, J=8.5 Hz, 2H, ArH), 7.21 (d, J=8.5 $\mathrm{Hz}, 2 \mathrm{H}, \mathrm{ArH}), 5.04\left(\mathrm{~s}, 2 \mathrm{H}, \mathrm{CH}_{2}\right), 3.99\left(\mathrm{t}, \mathrm{J}=6 \mathrm{~Hz}, 2 \mathrm{H}, \mathrm{CH}_{2}\right), 3.76\left(\mathrm{~s}, 2 \mathrm{H}, \mathrm{CH}_{2}\right), 3.56\left(\mathrm{~s}, 2 \mathrm{H}, \mathrm{CH}_{2}\right), 3.09$ (s, $\left.6 \mathrm{H}, 2 \times \mathrm{CH}_{3}\right), 2.93\left(\mathrm{t}, \mathrm{J}=6 \mathrm{~Hz}, 2 \mathrm{H}, \mathrm{CH}_{2}\right) \cdot{ }^{13} \mathrm{C} \mathrm{NMR}\left(\mathrm{CDCl}_{3}\right) \delta 143.45(\operatorname{ArC}), 137.32(\operatorname{ArC}), 135.97(\operatorname{ArC})$, $134.50(\mathrm{ArCH}), 133.75(\mathrm{ArC}), 132.44(\mathrm{ArCH}), 130.69(\mathrm{ArCH}), 129.89(\mathrm{ArCH}), 129.46(\mathrm{ArCH}), 128.92$ $(\mathrm{ArCH}), 125.59(\mathrm{ArC}), 118.56(\mathrm{ArC}), 111.52(\mathrm{ArC}), 67.26\left(\mathrm{CH}_{2}\right), 60.65\left(\mathrm{CH}_{2}\right), 58.31\left(\mathrm{CH}_{2}\right), 57.94\left(\mathrm{CH}_{2}\right)$, $49.33\left(2 \times \mathrm{CH}_{3}\right), 47.26\left(\mathrm{CH}_{2}\right)$. MS [ESI, $\left.\mathrm{m} / \mathrm{z}\right]$ : calcd for $\mathrm{C}_{26} \mathrm{H}_{28} \mathrm{Cl}_{2} \mathrm{~N}_{3}\left[\mathrm{M}^{+}\right], 452.1660$, found 452.1647 .

3-(2-(Dimethylamino)ethylamino)isoindolin-1-one (8) yield $93 \%$. To a solution of 2-formyl benzonitrile $(7 ; 1.5 \mathrm{mmol})$ in $\mathrm{MeOH}(25 \mathrm{~mL})$ was added dropwise 2-dimethylaminoethylamine $(2 ; 1.8$ $\mathrm{mmol}$ ). The solution was stirred at room temperature for $4 \mathrm{~h}$, sodium triacetoxyborohydride $(2.4$ $\mathrm{mmol}$ ) was added to the mixture and stirred at room temperature for $24 \mathrm{~h}$. The progress of the reaction was monitored by TLC. The solution was then dried in vacuo, and the crude residue was purified by column chromatography eluting with $\mathrm{CH}_{2} \mathrm{Cl}_{2} / \mathrm{CH}_{3} \mathrm{OH}$ 90:10. ${ }^{1} \mathrm{H}$ NMR $\left(\mathrm{CDCl}_{3}\right) \delta 7.78,7.76$ (2t, J=1 Hz, 1H, ArH), 7.70-7.69 (2m, 1H, ArH), 7.67 (ddd, J=7.5, 7, $1 \mathrm{~Hz}, 1 \mathrm{H}, \operatorname{ArH}), 7.55(\mathrm{~m}, 1 \mathrm{H}, \operatorname{ArH})$, $5.53(\mathrm{~s}, 1 \mathrm{H}, \mathrm{CH}), 3.34,3.33\left(2 \mathrm{t}, \mathrm{J}=1.5 \mathrm{~Hz}, 1 \mathrm{H}, \mathrm{CH}_{2}\right), 2.77,2.75\left(2 \mathrm{t}, \mathrm{J}=6.5 \mathrm{~Hz}, 1 \mathrm{H}, \mathrm{CH}_{2}\right), 2.62,2.59$ (2t, $\left.J=6.5 \mathrm{~Hz}, 1 \mathrm{H}, \mathrm{CH}_{2}\right), 2.46,2.45\left(2 \mathrm{t}, J=6.5 \mathrm{~Hz}, 2 \mathrm{H}, \mathrm{CH}_{2}\right), 2.25\left(\mathrm{~s}, 6 \mathrm{H}, 2 \mathrm{CH}_{3}\right) ;{ }^{13} \mathrm{C} \mathrm{NMR}\left(\mathrm{CDCl}_{3}\right) \delta 170.00$ $(C=0), 145.57(\operatorname{ArC}), 132.58(\operatorname{ArC}), 132.03(\mathrm{ArCH}), 129.09(\mathrm{ArCH}), 123.62(\mathrm{ArCH}), 123.41(\mathrm{ArCH}), 70.68$ $(\mathrm{CH}), 60.07\left(\mathrm{CH}_{2}\right), 45.28\left(2 \times \mathrm{CH}_{3}\right), 41.90\left(\mathrm{CH}_{2}\right) . \mathrm{MS}[\mathrm{ESI}, \mathrm{m} / \mathrm{z}]: 220.16[\mathrm{M}+\mathrm{H}], 242.14[\mathrm{M}+\mathrm{Na}]$.

$\mathbf{N}$-(4-chlorobenzyl)pyridin-2-amine (11a) yield $96 \%$. The solution of pyridin-2-amine (9a; $1.5 \mathrm{mmol})$ and 4-chlorobenzaldehyde (10a; $1.5 \mathrm{mmol})$ in $\mathrm{AcOH}(10 \mathrm{~mL})$ was stirred at room temperature for $4 \mathrm{~h}$, sodium triacetoxyborohydride $(2.4 \mathrm{mmol})$ was added to the mixture and stirred at room temperature for $24 \mathrm{~h}$. Water $\left(10 \mathrm{~mL}\right.$ ) was added and the mixture neutralized using $\mathrm{NaHCO}_{3}$ (sat. aq., $10 \mathrm{~mL})$. The organic layer was extracted with DCM $(2 \times 10 \mathrm{~mL})$, dried, evaporated and the crude residue was purified by column chromatography eluting with $\mathrm{CH}_{2} \mathrm{Cl}_{2} / \mathrm{CH}_{3} \mathrm{OH} 90: 10 .{ }^{1} \mathrm{H} \mathrm{NMR}\left(\mathrm{CDCl}_{3}\right) \delta$ $8.00(\mathrm{dd}, J=5,2 \mathrm{~Hz}, 1 \mathrm{H}, \operatorname{ArH}), 7.33$ (ddd, J= 8.5, 7, $2 \mathrm{~Hz}, 1 \mathrm{H}, \operatorname{Ar} H$ ), 7.15-7.25 (m, 4H, ArH), 6.52 (ddd, 
$J=7,5,1 \mathrm{~Hz}, 1 \mathrm{H}, \operatorname{Ar} H), 6.27(\mathrm{~d}, J=8.5 \mathrm{~Hz}, 1 \mathrm{H}, \mathrm{ArH}), 5.23$ (bs, $1 \mathrm{H}, \mathrm{NH}), 4.40\left(\mathrm{~s}, 2 \mathrm{H}, \mathrm{CH}_{2}\right) .{ }^{13} \mathrm{C}$ NMR $\left(\mathrm{CDCl}_{3}\right) \delta 158.37(\mathrm{ArC}), 147.76(\mathrm{ArCH}), 137.81(\mathrm{ArCH}), 137.72(\mathrm{ArC}), 132.93(\mathrm{ArC}), 128.76(\mathrm{ArCH})$, $128.63(\mathrm{ArCH}), 113.30(\mathrm{ArCH}), 106.91(\mathrm{ArCH}), 45.57\left(\mathrm{CH}_{2}\right) .[\mathrm{ESI}, \mathrm{m} / \mathrm{z}]: 219.1[\mathrm{M}+\mathrm{H}], 241.1[\mathrm{M}+\mathrm{Na}]$.

\section{General method for the preparation of intermediates $11(b-h)$ and 14}

The solution of the corresponding aniline derivatives $(9 \mathbf{b}-\mathbf{h} ; 1.5 \mathrm{mmol})$ and 4 -chlorobenzaldehyde (10a) or 4-fluorobenzaldehyde (10b); $(1.5 \mathrm{mmol})$ in dichloroethanol (DCE, $10 \mathrm{~mL}$ ) was stirred at reflux temperature for $8 \mathrm{~h}$. After cooling, sodium triacetoxyborohydride $(2.4 \mathrm{mmol})$ was added to the mixture and stirred at room temperature for $24 \mathrm{~h}$. Water $(10 \mathrm{~mL})$ was added. The organic layer was extracted with DCM $(2 \times 10 \mathrm{~mL})$, dried, evaporated and the crude residue was purified by column chromatography eluting with $n$-hexane/EtOAc 90:10.

Methyl 4-((4-chlorobenzyl)amino)-2-hydroxybenzoate (11b) yield $97 \% .{ }^{1} \mathrm{H}$ NMR $\left(\mathrm{CDCl}_{3}\right) \delta 10.99$ (s, $1 \mathrm{H}, \mathrm{OH}$ ), $7.63(\mathrm{~d}, J=9 \mathrm{~Hz}, 1 \mathrm{H}, \operatorname{ArH}), 7.34(\mathrm{~d}, J=8.5 \mathrm{~Hz}, 2 \mathrm{H}, \operatorname{ArH}), 7.29$ (d, J = 8.5 Hz, 2H, ArH), 6.14 (dd, $J=8.5,2 \mathrm{~Hz}, 1 \mathrm{H}, \operatorname{ArH}), 6.11(\mathrm{~d}, J=2 \mathrm{~Hz}, 1 \mathrm{H}, \operatorname{ArH}), 4.54$ (bs, $1 \mathrm{H}, \mathrm{NH}), 4.37\left(\mathrm{~s}, 2 \mathrm{H}, \mathrm{CH}_{2}\right), 3.90(\mathrm{~s}, 3 \mathrm{H}$, $\left.\mathrm{CH}_{3}\right) ;{ }^{13} \mathrm{C} \mathrm{NMR}\left(\mathrm{CDCl}_{3}\right) \delta 170.49(\mathrm{C}=\mathrm{O}), 163.77(\operatorname{ArC}), 153.86(\operatorname{ArC}), 136.68(\operatorname{ArC}), 133.34(\operatorname{ArCH})$, 131.29 ( $\mathrm{ArCH}), 128.95(\mathrm{ArCH}), 128.62(\mathrm{ArCH}), 105.63(\mathrm{ArCH}), 102.38(\mathrm{ArC}), 98.20(\mathrm{ArCH}), 51.64\left(\mathrm{CH}_{3}\right)$, $46.85\left(\mathrm{CH}_{2}\right) . \mathrm{MS}[\mathrm{ESI}, \mathrm{m} / \mathrm{z}]: 292.1[\mathrm{M}+\mathrm{H}]$.

Methyl 4-((4-chlorobenzyl)amino)-2-hydroxybenzoate (11c) yield $98 \% .{ }^{1} \mathrm{H}$ NMR $\left(\mathrm{CDCl}_{3}\right) \delta 10.21$ (s, $\mathrm{OH})$, 7.36-7.30 (m, 4H, ArH), $7.08(\mathrm{dd}, J=2.5,1 \mathrm{~Hz}, 1 \mathrm{H}, \operatorname{ArH}), 6.89-6.86(\mathrm{~m}, 2 \mathrm{H}, \operatorname{ArH}), 4.29(\mathrm{~s}, 2 \mathrm{H}$, $\left.\mathrm{CH}_{2}\right), 3.94\left(\mathrm{~s}, 3 \mathrm{H}, \mathrm{CH}_{3}\right) ;{ }^{13} \mathrm{C} \mathrm{NMR}\left(\mathrm{CDCl}_{3}\right) \delta 170.48(\mathrm{C}=\mathrm{O}), 154.35(\operatorname{ArC}), 140.56(\operatorname{ArC}), 137.92(\operatorname{ArC})$, $132.87(\mathrm{ArC}), 128.83(\mathrm{ArCH}), 128.70(\mathrm{ArCH}), 122.76(\mathrm{ArCH}), 118.20(\mathrm{ArCH}), 112.21(\mathrm{ArC}), 111.75$ $(\mathrm{ArCH}), 52.17\left(\mathrm{CH}_{3}\right), 48.33\left(\mathrm{CH}_{2}\right) . \mathrm{MS}[\mathrm{ESI}, \mathrm{m} / \mathrm{z}]: 292.1[\mathrm{M}+\mathrm{H}]$.

Methyl 4-((4-chlorobenzyl)amino)-2-methoxybenzoate (11d) yield $85 \% .{ }^{1} \mathrm{H}$ NMR $\left(\mathrm{CDCl}_{3}\right) \delta 7.78$ (d, $J=8.5 \mathrm{~Hz}, 1 \mathrm{H}, \operatorname{ArH}), 7.35$ (d, J=8.5 Hz, 2H, ArH), 7.31 (d, J=9 Hz, 2H, ArH), 7.21 (dd, J=8.5, $2 \mathrm{~Hz}, 1 \mathrm{H}$, $\operatorname{ArH}), 6.13(\mathrm{~d}, J=2 \mathrm{~Hz}, 1 \mathrm{H}, \mathrm{ArH}), 4.51(\mathrm{t}, J=5.5 \mathrm{~Hz}, 1 \mathrm{H}, \mathrm{NH}), 4.39\left(\mathrm{~d}, \mathrm{~J}=5.5 \mathrm{~Hz}, 2 \mathrm{H}, \mathrm{CH}_{2}\right), 3.85\left(\mathrm{~s}, 3 \mathrm{H}, \mathrm{CH}_{3}\right)$, $3.84\left(\mathrm{~s}, 3 \mathrm{H}, \mathrm{CH}_{3}\right) ;{ }^{13} \mathrm{C}$ NMR $\left(\mathrm{CDCl}_{3}\right) \delta 166.22(\mathrm{C}=\mathrm{O}), 161.79(\operatorname{ArC}), 152.66(\operatorname{ArC}), 136.86(\operatorname{ArC}), 134.17$ $(\mathrm{ArCH}), 133.33(\mathrm{ArC}), 128.95(\mathrm{ArCH}), 128.67(\mathrm{ArCH}), 108.43(\mathrm{ArC}), 104.40(\mathrm{ArCH}), 95.81(\mathrm{ArCH}), 55.76$ $\left(\mathrm{CH}_{3}\right), 51.41\left(\mathrm{CH}_{3}\right), 47.12\left(\mathrm{CH}_{2}\right)$. MS [ESI, m/z]: $306.1[\mathrm{M}+\mathrm{H}], 328.1[\mathrm{M}+\mathrm{Na}]$.

4-((4-Chlorobenzyl)amino)-2-methoxybenzoic acid (11e) yield $96 \%{ }^{1} \mathrm{H} N M R\left(\mathrm{CDCl}_{3}\right) \delta 10.25$ (bs, $1 \mathrm{H}$, $\mathrm{COOH}$ ), $7.97(\mathrm{~d}, J=8.5 \mathrm{~Hz}, 1 \mathrm{H}, \operatorname{ArH}), 7.36(\mathrm{~d}, J=8.5 \mathrm{~Hz}, 2 \mathrm{H}, \operatorname{ArH}), 7.30$ (d, J=8.5 Hz, 2H, ArH), 6.34 (dd, $J=8.5,2 \mathrm{~Hz}, 1 \mathrm{H}, \mathrm{ArH}), 6.14(\mathrm{~d}, \mathrm{~J}=2.5 \mathrm{~Hz}, 1 \mathrm{H}, \mathrm{ArH}), 4.81$ (bs, $1 \mathrm{H}, \mathrm{NH}), 4.41\left(\mathrm{~s}, 2 \mathrm{H}, \mathrm{CH}_{2}\right), 3.96\left(\mathrm{~s}, 3 \mathrm{H}, \mathrm{CH}_{3}\right)$;

${ }^{13} \mathrm{C}$ NMR $\left(\mathrm{CDCl}_{3}\right) \delta 165.87(\mathrm{C}=\mathrm{O}), 159.98(\operatorname{ArC}), 153.52(\operatorname{ArC}), 136.41(\operatorname{ArC}), 135.40(\mathrm{ArCH}), 133.49$ ( $\mathrm{ArC}), 129.05(\mathrm{ArCH}), 128.60(\mathrm{ArCH}), 106.47(\mathrm{ArCH}), 106.41(\mathrm{ArC}), 94.64(\mathrm{ArCH}), 56.26\left(\mathrm{CH}_{3}\right), 47.01$ $\left(\mathrm{CH}_{2}\right) . \mathrm{MS}[\mathrm{ESI}, \mathrm{m} / \mathrm{z}]: 292.1[\mathrm{M}+\mathrm{H}], 314.1[\mathrm{M}+\mathrm{Na}]$. 
4-(4-Chlorobenzylamino)-2-hydroxybenzoic acid (11f) yield $81 \% .{ }^{1} \mathrm{H}$ NMR (DMSO- $\left.d_{6}\right) \delta 12.87$ (bs, $1 \mathrm{H}, \mathrm{COOH}), 11.44$ (bs, $1 \mathrm{H}, \mathrm{OH}), 7.45(\mathrm{~d}, J=9 \mathrm{~Hz}, 1 \mathrm{H}, \operatorname{ArH}), 7.40(\mathrm{~d}, J=8.5 \mathrm{~Hz}, 2 \mathrm{H}, \operatorname{ArH}), 7.35(\mathrm{~d}, J=8.5$ $\mathrm{Hz}, 2 \mathrm{H}, \operatorname{ArH}), 7.20(\mathrm{t}, J=6 \mathrm{~Hz}, 1 \mathrm{H}, \mathrm{NH}), 6.19(\mathrm{dd}, J=8.5,2 \mathrm{~Hz}, 1 \mathrm{H}, \operatorname{ArH}), 6.93(\mathrm{~d}, J=2 \mathrm{~Hz}, 1 \mathrm{H}, \operatorname{ArH}), 4.32$ $\left(\mathrm{d}, J=6 \mathrm{~Hz}, 2 \mathrm{H}, \mathrm{CH}_{2}\right) ;{ }^{13} \mathrm{C}$ NMR (DMSO-d $\left.d_{6}\right) \delta 172.55(C=0), 163.95(\operatorname{ArC}), 155.19(\operatorname{ArC}), 138.91(\operatorname{ArC})$, $131.80(\mathrm{ArC}), 131.59(\mathrm{ArCH}), 129.41(\mathrm{ArCH}), 128.82(\mathrm{ArCH}), 106.05(\mathrm{ArCH}), 100.90(\operatorname{ArC}), 97.32$ $(\mathrm{ArCH}), 45.46\left(\mathrm{CH}_{2}\right)$. MS [ESI, m/z]: $278.1[\mathrm{M}+\mathrm{H}], 300.0[\mathrm{M}+\mathrm{Na}]$. anal. calcd for $\mathrm{C}_{14} \mathrm{H}_{12} \mathrm{ClNO}_{3}: \mathrm{C}, 60.55$; $H, 4.36 ; N, 5.04$. Found: $C, 60.18 ; H, 4.39 ; N, 4.95$.

5-((4-Chlorobenzyl)amino)-2-methoxybenzoic acid (11g) yield $60 \% .{ }^{1} \mathrm{H} \mathrm{NMR}\left(\mathrm{CDCl}_{3}\right) \delta 10.90$ (bs, $1 \mathrm{H}$, $\mathrm{COOH}), 7.48(\mathrm{~d}, J=3 \mathrm{~Hz}, 1 \mathrm{H}, \operatorname{ArH}), 7.33(\mathrm{~d}, J=8.5 \mathrm{~Hz}, 2 \mathrm{H}, \operatorname{ArH}), 7.30$ (d, J=8.5 Hz, 2H, ArH), 6.92 (d, J= $9 \mathrm{~Hz}, 1 \mathrm{H}, \mathrm{ArH}), 6.79(\mathrm{dd}, \mathrm{J}=9 \mathrm{~Hz}, 3 \mathrm{~Hz}, 1 \mathrm{H}, \mathrm{ArH}), 4.34\left(\mathrm{~s}, 2 \mathrm{H}, \mathrm{CH}_{2}\right), 4.01\left(\mathrm{~s}, 3 \mathrm{H}, \mathrm{CH}_{3}\right) .{ }^{13} \mathrm{CNMR}\left(\mathrm{CDCl}_{3}\right) \delta$ $165.55(C=0), 150.55(\operatorname{ArC}), 142.80(\operatorname{ArC}), 137.25(\operatorname{ArC}), 133.15(\operatorname{ArC}), 128.84(\mathrm{ArCH}), 128.75(\operatorname{ArCH})$, $119.24(\mathrm{ArCH}), 118.19(\mathrm{ArC}), 117.05(\mathrm{ArCH}), 113.30(\mathrm{ArCH}), 57.22\left(\mathrm{CH}_{3}\right), 48.00\left(\mathrm{CH}_{2}\right) . \operatorname{MS}[\mathrm{AP}, \mathrm{m} / \mathrm{z}]$ : $292.1[\mathrm{M}+\mathrm{H}]$.

Methyl 4-((4-chlorobenzyl)amino)benzoate (11h) yield $95 \% .{ }^{1} \mathrm{H} N M R\left(\mathrm{CDCl}_{3}\right) \delta 7.88(\mathrm{~d}, \mathrm{~J}=9 \mathrm{~Hz}, 2 \mathrm{H}$, $\operatorname{ArH}), 7.34(\mathrm{~d}, J=9 \mathrm{~Hz}, 2 \mathrm{H}, \operatorname{ArH}), 7.30(\mathrm{~d}, J=9 \mathrm{~Hz}, 2 \mathrm{H}, \operatorname{ArH}), 6.59$ (d, J= $9 \mathrm{~Hz}, 2 \mathrm{H}, \operatorname{ArH}), 4.39\left(\mathrm{~s}, 2 \mathrm{H}, \mathrm{CH}_{2}\right)$, $3.87\left(\mathrm{~s}, 3 \mathrm{H}, \mathrm{CH}_{3}\right) .{ }^{13} \mathrm{C}$ NMR $\left(\mathrm{CDCl}_{3}\right) \delta 167.21(\mathrm{C}=\mathrm{O}), 151.46(\operatorname{ArC}), 136.93(\operatorname{ArC}), 133.27(\operatorname{ArC}), 133.27$ ( $\mathrm{ArCH}), 129.48(\mathrm{ArCH}), 129.22(\mathrm{ArCH}), 119.03(\mathrm{ArC}), 111.74(\mathrm{ArCH}), 51.59\left(\mathrm{CH}_{3}\right), 47.01\left(\mathrm{CH}_{2}\right) . \mathrm{MS}[\mathrm{ESI}$, $\mathrm{m} / \mathrm{z}]: 274.1[\mathrm{M}-\mathrm{H}]$.

\section{General method for the preparation of intermediates $11 \mathrm{i}-\mathrm{k}$}

The solution of the corresponding aniline $(9 \mathbf{i}-\mathbf{k} ; 1.5 \mathrm{mmol})$ and 4-chlorobenzaldehyde or 4fluorobenzaldehyde $(10 \mathrm{a} ; 1.5 \mathrm{mmol})$ in $\mathrm{AcOH}(10 \mathrm{~mL})$ was stirred at room temperature for $4 \mathrm{~h}$, sodium triacetoxyborohydride $(2.4 \mathrm{mmol})$ was added to the mixture and stirred at room temperature for $24 \mathrm{~h}$. Water $\left(10 \mathrm{~mL}\right.$ ) was added and the mixture neutralized using $\mathrm{NaHCO}_{3}$ (sat. aq., $10 \mathrm{~mL})$. The organic layer was extracted with DCM $(2 \times 10 \mathrm{~mL})$, dried and evaporated.

2-((4-Chlorobenzyl)amino)benzonitrile (11i) yield $95 \%$. ${ }^{1} \mathrm{H} \mathrm{NMR}\left(\mathrm{CDCl}_{3}\right) \delta 7.44$ (d, J= $7.5 \mathrm{~Hz}, 1 \mathrm{H}$, $\operatorname{ArH})$, 7.34-7.37 (m, 3H, ArH), $7.30(\mathrm{~d}, J=9 \mathrm{~Hz}, 2 \mathrm{H}, \operatorname{ArH}), 6.73(\mathrm{t}, J=6.5 \mathrm{~Hz}, 1 \mathrm{H}, \operatorname{ArH}), 6.59(\mathrm{~d}, J=8.5 \mathrm{~Hz}$, $1 \mathrm{H}, \mathrm{ArH}), 5.05(\mathrm{t}, J=6 \mathrm{~Hz}, 1 \mathrm{H}, \mathrm{NH}), 4.44\left(\mathrm{~d}, J=6 \mathrm{~Hz}, 2 \mathrm{H}, \mathrm{CH}_{2}\right) .{ }^{13} \mathrm{C} \mathrm{NMR}\left(\mathrm{CDCl}_{3}\right) \delta 149.80(\mathrm{CN}), 136.28$ $(\mathrm{ArCH}), 134.30(\mathrm{ArC}), 133.42(\mathrm{ArC}), 132.81(\mathrm{ArCH}), 129.05(\mathrm{ArCH}), 128.41(\mathrm{ArCH}), 117.77(\operatorname{ArC})$, 117.19 ( $\mathrm{ArCH}), 111.04(\mathrm{ArCH}), 96.18(\mathrm{ArC}), 46.84\left(\mathrm{CH}_{2}\right) . \mathrm{MS}[\mathrm{EI}, \mathrm{m} / \mathrm{z}]: 242.061$.

3-((4-Chlorobenzyl)amino)benzonitrile (11j) yield $97 \% .{ }^{1} \mathrm{H} \mathrm{NMR}\left(\mathrm{CDCl}_{3}\right) \delta 7.36(\mathrm{~d}, \mathrm{~J}=8.5 \mathrm{~Hz}, 2 \mathrm{H}, \mathrm{ArH})$, 7.30 (d, J=8.5 Hz, 2H, ArH), 7.29 (td, J=7.5, $1 \mathrm{~Hz}, 1 \mathrm{H}, \operatorname{ArH}), 7.01$ (dt, J= 7.5, 1.5 Hz, 1H, ArH), 6.81 (m, $1 \mathrm{H}, \operatorname{Ar} H), 4.34\left(\mathrm{~d}, \mathrm{~J}=5 \mathrm{~Hz}, 2 \mathrm{H}, \mathrm{CH}_{2}\right) ;{ }^{13} \mathrm{C}$ NMR $\left(\mathrm{CDCl}_{3}\right) \delta 147.84(\operatorname{ArC}), 136.56(\operatorname{ArC}), 133.41(\operatorname{ArC})$, $130.00(\mathrm{ArCH}), 129.01(\mathrm{ArCH}), 128.62(\mathrm{ArCH}), 121.38(\mathrm{ArCH}), 119.31(\mathrm{CN}), 117.41(\mathrm{ArCH}), 115.26$ $(\operatorname{ArCH}), 113.07(\operatorname{ArC}), 47.31\left(\mathrm{CH}_{2}\right) . \mathrm{MS}[\mathrm{ESI}, \mathrm{m} / \mathrm{z}]: 242.0610[\mathrm{M}+\mathrm{H}]$. 
4-((4-Chlorobenzyl)amino)benzonitrile (11k) yield $96 \% .{ }^{1} \mathrm{H} N M R\left(\mathrm{CDCl}_{3}\right) \delta 7.45(\mathrm{~d}, \mathrm{~J}=9 \mathrm{~Hz}, 2 \mathrm{H}, \mathrm{ArH})$, 7.36 (d, J=8.5 Hz, 2H, ArH), 7.29 (d, J=8.5 Hz, 2H, ArH), 6.60 (d, J=9 Hz, 2H, ArH), 4.63 (bs, $1 \mathrm{H}, N H)$, $4.39\left(\mathrm{~d}, J=5 \mathrm{~Hz}, 2 \mathrm{H}, \mathrm{CH}_{2}\right), 4.39\left(\mathrm{~d}, J=5.5 \mathrm{~Hz}, 2 \mathrm{H}, \mathrm{CH}_{2}\right), 3.87\left(\mathrm{~s}, 3 \mathrm{H}, \mathrm{CH}_{3}\right), 3.84\left(\mathrm{~s}, 3 \mathrm{H}, \mathrm{CH}_{3}\right) ;{ }^{13} \mathrm{C} \mathrm{NMR}$ $\left(\mathrm{CDCl}_{3}\right) \delta 150.89(\mathrm{ArC}), 136.39(\mathrm{ArC}), 133.76(\mathrm{ArCH}), 133.44(\operatorname{ArC}), 129.35(\mathrm{ArCH}), 128.56(\operatorname{ArCH})$, $120.29(\mathrm{CN}), 112.51(\mathrm{ArCH}), 99.42(\mathrm{ArC}), 46.83\left(\mathrm{CH}_{2}\right) . \mathrm{MS}[\mathrm{ESI}, \mathrm{m} / \mathrm{z}]: 242.06[\mathrm{M}+\mathrm{H}]$.

3-(4-Fluorobenzylamino)benzonitrile (11) yield $57 \%$. The solution of 3-aminobenzonitrile (9l; 1.5 $\mathrm{mmol}$ ) and 4-fluorobenzaldehyde (10b; $1.5 \mathrm{mmol})$ in $\mathrm{MeOH}(10 \mathrm{~mL})$ was stirred at room temperature for $7 \mathrm{~h}$, sodium triacetoxyborohydride $(2.4 \mathrm{mmol})$ was added to the mixture and stirred at room temperature for $24 \mathrm{~h}$. Water $(10 \mathrm{~mL})$ was added to the mixture and the product was extracted using ethyl acetate and water and recrystallised from methanol. ${ }^{1} \mathrm{H}-\mathrm{NMR}\left(\mathrm{CDCl}_{3}\right) \delta 7.22$ (dd, $J=9,5 \mathrm{~Hz}, 2 \mathrm{H}, \operatorname{ArH}), 7.12$ (dd, J= 9, $7.5 \mathrm{~Hz}, 1 \mathrm{H}, \operatorname{ArH}), 6.95$ (t, J=9 Hz, 2H, ArH), 6.87 (d, J= $8 \mathrm{~Hz}, 1 \mathrm{H}$, $\operatorname{ArH}), 6.72-6.70(\mathrm{~m}, 2 \mathrm{H}, \mathrm{ArH}), 4.21(\mathrm{~s}, 2 \mathrm{H}, \mathrm{CH} 2), 4.37(\mathrm{~s}, 1 \mathrm{H}, \mathrm{NH}) . \mathrm{MS}[\mathrm{ESI}, \mathrm{m} / \mathrm{z}]:[\mathrm{M}+\mathrm{H}] 227.1$ \& $[\mathrm{M}+\mathrm{Na}] 249.1$.

\section{General method for the preparation of compounds 13a-I}

The mixture of the corresponding secondary amine (11a-l; $1.5 \mathrm{mmol})$, dimethylaminoacetyl chloride hydrochloride (12; $1.6 \mathrm{mmol})$ and potassium carbonate $(3.2 \mathrm{mmol})$ in THF $(10 \mathrm{~mL})$ was stirred at room temperature for $24 \mathrm{~h}$. The mixture was extracted with EtOAc $(3 \times 20 \mathrm{~mL})$, the organic layer was dried, evaporated and the crude residue was purified by column chromatography eluting with; $\mathrm{CH}_{2} \mathrm{Cl}_{2} / \mathrm{CH}_{3} \mathrm{OH}$ 90:10.

$\mathbf{N}$-(4-chlorobenzyl)-2-(dimethylamino)- $\mathbf{N}$-(pyridin-2-yl) acetamide (13a) yield $89 \% .{ }^{1} \mathrm{H} N M R\left(\mathrm{CDCl}_{3}\right)$ $\delta 8.40$ (dd, J=5.0, $1.0 \mathrm{~Hz}, 1 \mathrm{H}, \operatorname{ArH}$ ), 7.59 (ddd, J=8.0, 7.5, $2 \mathrm{~Hz}, 1 \mathrm{H}, \operatorname{ArH}$ ), 7.15 (d, J=8.5 Hz, 2H, $\operatorname{ArH}$ ), 7.13-7.09 (m, 3H, $\mathrm{ArH}), 7.08-7.00(\mathrm{~m}, 1 \mathrm{H}, \mathrm{ArH}), 5.00\left(\mathrm{~s}, 2 \mathrm{H}, \mathrm{CH}_{2}\right), 3.06\left(\mathrm{~s}, 2 \mathrm{H}, \mathrm{CH}_{2}\right), 2.19(\mathrm{~s}, 6 \mathrm{H}$, $\left.2 \times \mathrm{CH}_{3}\right) .{ }^{13} \mathrm{C} \mathrm{NMR}\left(\mathrm{CDCl}_{3}\right) \delta 170.09(\mathrm{C}=\mathrm{O}), 154.60(\mathrm{ArC}), 149.16(\mathrm{ArCH}), 138.14(\mathrm{ArCH}), 136.12(\mathrm{ArC})$, 133.07 ( $\mathrm{ArC}), 129.43(\mathrm{ArCH}), 128.76(\mathrm{ArCH}), 122.19(\mathrm{ArCH}), 121.27(\mathrm{ArCH}), 61.59\left(\mathrm{CH}_{2}\right), 50.52\left(\mathrm{CH}_{2}\right)$, $45.30\left(2 \times \mathrm{CH}_{3}\right)$. [ESI, m/z]: $304.1212[\mathrm{M}+\mathrm{H}], 326.1030[\mathrm{M}+\mathrm{Na}]$. calcd for $\mathrm{C}_{16} \mathrm{H}_{18} \mathrm{ClN}_{3} \mathrm{O}[\mathrm{M}+\mathrm{H}], 304.1211$; found, 304.1212 .

Methyl 4-(N-(4-chlorobenzyl)-2-(dimethylamino)acetamido)-2-hydroxy benzoate (13b) yield $88 \%$. ${ }^{1} \mathrm{H}$ NMR $\left(\mathrm{CDCl}_{3}\right) \delta 10.86$ (bs, 1H, OH), 7.82 (d, J = 8.5 Hz, 1H, ArH), 7.25 (d, J = 8.5 Hz, 2H, ArH), 7.16 $(\mathrm{d}, J=8.5 \mathrm{~Hz}, 2 \mathrm{H}, \operatorname{ArH}), 6.71(\mathrm{~d}, J=2 \mathrm{~Hz}, 1 \mathrm{H}, \operatorname{ArH}), 6.53(\mathrm{dd}, J=8.5,2 \mathrm{~Hz}, 1 \mathrm{H}, \operatorname{ArH}), 4.88\left(\mathrm{~s}, 2 \mathrm{H}, \mathrm{CH}_{2}\right)$, $3.98\left(\mathrm{~s}, 3 \mathrm{H}, \mathrm{CH}_{3}\right), 2.97\left(\mathrm{~s}, 2 \mathrm{H}, \mathrm{CH}_{2}\right), 2.28\left(\mathrm{~s}, 6 \mathrm{H}, 2 \times \mathrm{CH}_{3}\right) ;{ }^{13} \mathrm{CNMR}\left(\mathrm{CDCl}_{3}\right) \delta 169.84(\mathrm{C}=\mathrm{O}), 169.32(\mathrm{C}=\mathrm{O})$, $162.36(\operatorname{ArC}), 148.09(\operatorname{ArC}), 135.60(\operatorname{ArC}), 133.45(\operatorname{ArC}), 131.11(\mathrm{ArCH}), 130.05(\mathrm{ArCH}), 128.67(\operatorname{ArCH})$, $119.10(\mathrm{ArCH}), 116.90(\mathrm{ArCH}), 111.99(\mathrm{ArC}), 60.90\left(\mathrm{CH}_{2}\right), 52.56\left(\mathrm{OCH}_{3}\right), 54.10\left(\mathrm{CH}_{2}\right), 45.56\left(2 \times \mathrm{CH}_{3}\right)$. MS [ESI, m/z]: calcd for $\mathrm{C}_{19} \mathrm{H}_{21} \mathrm{ClN}_{2} \mathrm{O}_{4}[\mathrm{M}+\mathrm{H}], 377.1268$; found, 377.1266 .

Methyl 5-(N-(4-chlorobenzyl)-2-(dimethylamino)acetamido)-2-hydroxy benzoate (13c) yield $92 \%$. 
${ }^{1} \mathrm{H} \mathrm{NMR}\left(\mathrm{CDCl}_{3}\right) \delta 10.83$ (bs, $\left.1 \mathrm{H}, \mathrm{OH}\right), 7.47(\mathrm{~d}, J=2.5 \mathrm{~Hz}, 1 \mathrm{H}, \mathrm{ArH}), 7.26(\mathrm{~d}, J=8.5 \mathrm{~Hz}, 2 \mathrm{H}, \mathrm{ArH}), 7.15$ (d, $J=8.5 \mathrm{~Hz}, 2 \mathrm{H}, \operatorname{ArH}), 7.01(\mathrm{dd}, J=9.0,2.5 \mathrm{~Hz}, 1 \mathrm{H}, \operatorname{ArH}), 6.94(\mathrm{~d}, J=9 \mathrm{~Hz}, 1 \mathrm{H}, \operatorname{ArH}), 4.82\left(\mathrm{~s}, 2 \mathrm{H}, \mathrm{CH}_{2}\right.$ ), $3.97\left(\mathrm{~s}, 3 \mathrm{H}, \mathrm{CH}_{3}\right), 2.85\left(\mathrm{~s}, 2 \mathrm{H}, \mathrm{CH}_{2}\right), 2.26\left(\mathrm{~s}, 6 \mathrm{H}, 2 \mathrm{CH}_{3}\right) ;{ }^{13} \mathrm{C} \mathrm{NMR}\left(\mathrm{CDCl}_{3}\right) \delta 169.79(\mathrm{C}=\mathrm{O}), 169.67(\mathrm{C}=\mathrm{O})$, $161.15(\operatorname{ArC}), 136.00(\mathrm{ArCH}), 135.70(\operatorname{ArC}), 133.45(\operatorname{ArC}), 132.49(\operatorname{ArC}), 130.45(\mathrm{ArCH}), 129.53(\operatorname{ArCH})$, 128.65 (ArCH), $118.92(\mathrm{ArCH}), 112.90(\mathrm{ArC}), 60.70\left(\mathrm{CH}_{2}\right), 52.67\left(\mathrm{OCH}_{3}\right), 52.42\left(\mathrm{CH}_{2}\right), 45.61\left(2 \times \mathrm{CH}_{3}\right)$. MS [ESI, m/z]: calcd for $\mathrm{C}_{19} \mathrm{H}_{21} \mathrm{ClN}_{2} \mathrm{O}_{4}[\mathrm{M}+\mathrm{H}], 377.1268$; found, 377.1266 .

Methyl 4-(N-(4-chlorobenzyl)-2-(dimethylamino)acetamido)-2-methoxy benzoate (13d) yield $95 \%$. ${ }^{1} \mathrm{H}$ NMR $\left(\mathrm{CDCl}_{3}\right) \delta 7.78(\mathrm{~d}, J=8.5 \mathrm{~Hz}, 1 \mathrm{H}, \mathrm{ArH}), 7.27(\mathrm{~d}, J=8.5 \mathrm{~Hz}, 2 \mathrm{H}, \operatorname{ArH}), 7.17(\mathrm{~d}, J=8.5 \mathrm{~Hz}, 2 \mathrm{H}$, $\operatorname{ArH}), 6.65(\mathrm{dd}, J=8.5,2 \mathrm{~Hz}, 1 \mathrm{H}, \operatorname{ArH}), 6.55(\mathrm{~s}, 1 \mathrm{H}, \operatorname{ArH}), 4.87\left(\mathrm{~s}, 2 \mathrm{H}, \mathrm{CH}_{2}\right), 3.92\left(\mathrm{~s}, 3 \mathrm{H}, \mathrm{CH}_{3}\right), 3.78(\mathrm{~s}$, $\left.3 \mathrm{H}, \mathrm{CH}_{3}\right), 2.91\left(\mathrm{~s}, 2 \mathrm{H}, \mathrm{CH}_{2}\right), 2.27\left(\mathrm{~s}, 6 \mathrm{H}, 2 \times \mathrm{CH}_{3}\right) ;{ }^{13} \mathrm{C} \mathrm{NMR}\left(\mathrm{CDCl}_{3}\right) \delta 169.31(\mathrm{C}=0), 165.90(\mathrm{C}=0), 159.75$ $(\mathrm{ArC}), 146.06(\mathrm{ArC}), 135.76(\mathrm{ArC}), 133.57(\mathrm{ArC}), 132.70(\mathrm{ArCH}), 130.38(\mathrm{ArCH}), 128.67(\mathrm{ArCH}), 119.88$ $(\mathrm{ArCH}), 114.09(\mathrm{ArC}), 112.20(\mathrm{ArCH}), 60.87\left(\mathrm{CH}_{2}\right), 56.15\left(\mathrm{CH}_{3}\right), 52.27\left(\mathrm{CH}_{3}\right), 52.20\left(\mathrm{CH}_{2}\right), 45.68\left(2 \times \mathrm{CH}_{3}\right)$. MS [ESI, m/z]: calcd for $\mathrm{C}_{20} \mathrm{H}_{23} \mathrm{ClN}_{2} \mathrm{O}_{4}[\mathrm{M}+\mathrm{H}], 391.1425$; found, 391.1418 .

4-( $N$-(4-chlorobenzyl)-2-(dimethylamino)acetamido)-2-methoxybenzoic acid (13e) yield $79 \%{ }^{1} \mathrm{H}$ $\operatorname{NMR}\left(\mathrm{CDCl}_{3}\right) \delta 7.91(\mathrm{~d}, J=5.5 \mathrm{~Hz}, 1 \mathrm{H}, \operatorname{ArH}), 7.17(\mathrm{~d}, J=9 \mathrm{~Hz}, 2 \mathrm{H}, \operatorname{ArH}), 7.06(\mathrm{~d}, J=9 \mathrm{~Hz}, 2 \mathrm{H}, \operatorname{ArH}), 6.66$ (dd, J = 5.5, $1 \mathrm{~Hz}, 1 \mathrm{H}, \operatorname{ArH}), 6.52(\mathrm{~s}, 1 \mathrm{H}, \mathrm{ArH}), 4.78\left(\mathrm{~s}, 2 \mathrm{H}, \mathrm{CH}_{2}\right), 3.75\left(\mathrm{~s}, 3 \mathrm{H}, \mathrm{CH}_{3}\right), 2.97\left(\mathrm{~s}, 2 \mathrm{H}, \mathrm{CH}_{2}\right), 2.90$ $\left(\mathrm{s}, 6 \mathrm{H}, 2 \times \mathrm{CH}_{3}\right) ;{ }^{13} \mathrm{C} \mathrm{NMR}\left(\mathrm{CDCl}_{3}\right) \delta 168.91(\mathrm{C}=\mathrm{O}), 168.83(\mathrm{C}=\mathrm{O}), 159.71(\operatorname{ArC}), 142.67(\operatorname{ArC}), 137.93$ $(\operatorname{ArC}), 135.63(\operatorname{ArC}), 133.35(\operatorname{ArC}), 132.70(\mathrm{ArCH}), 131.44(\mathrm{ArCH}), 130.63(\mathrm{ArCH}), 122.06(\operatorname{ArCH})$, $113.67(\mathrm{ArCH}), 61.17\left(\mathrm{CH}_{2}\right), 57.19\left(\mathrm{OCH}_{3}\right), 54.20\left(\mathrm{CH}_{2}\right), 46.07\left(2 \times \mathrm{CH}_{3}\right) . \mathrm{MS}$ [ESI, m/z]: calcd for $\mathrm{C}_{19} \mathrm{H}_{21} \mathrm{ClN}_{2} \mathrm{O}_{4}[\mathrm{M}+\mathrm{H}], 377.1268$; found, 377.1266.

4-(N-(4-chlorobenzyl)-2-(dimethylamino)acetamido)-2-hydroxybenzoic acid (13f) yield $61 \%{ }^{1} \mathrm{H}$ NMR (DMSO-d $\left.d_{6}\right) \delta 7.65(\mathrm{~d}, J=8 \mathrm{~Hz}, 1 \mathrm{H}, \operatorname{ArH}), 7.38(\mathrm{~d}, J=8.5 \mathrm{~Hz}, 2 \mathrm{H}, \operatorname{ArH}), 7.25(\mathrm{~d}, J=8.5 \mathrm{~Hz}, 2 \mathrm{H}$, $\operatorname{ArH}), 6.48(\mathrm{~d}, J=2 \mathrm{~Hz}, 1 \mathrm{H}, \operatorname{ArH}), 6.41(\mathrm{dd}, J=8,1.5 \mathrm{~Hz}, 1 \mathrm{H}, \operatorname{ArH}), 4.86(\mathrm{~s}, 2 \mathrm{H}), 3.73(\mathrm{~s}, 2 \mathrm{H}), 2.62(\mathrm{~s}$, $6 H) ;{ }^{13} \mathrm{C}$ NMR (DMSO- $\left.d_{6}\right) \delta 170.79(C=0), 165.90(C=0), 164.79(\operatorname{ArC}), 142.76(\operatorname{ArC}), 136.43(\operatorname{ArC})$, $132.36(\mathrm{ArC}), 131.33(\mathrm{ArCH}), 130.38(\mathrm{ArCH}), 128.79(\mathrm{ArCH}), 120.81(\mathrm{ArC}), 115.75(\mathrm{ArCH}), 115.34$ $(\mathrm{ArCH}), 58.33\left(\mathrm{CH}_{2}\right), 51.93\left(\mathrm{CH}_{2}\right), 44.28\left(2 \times \mathrm{CH}_{3}\right) . \mathrm{MS}$ [ESI, m/z]: calcd for $\mathrm{C}_{18} \mathrm{H}_{19} \mathrm{ClN}_{2} \mathrm{O}_{4}[\mathrm{M}+\mathrm{H}]$, 363.1123; found, 363.1112 .

5-( $N$-(4-chlorobenzyl)-2-(dimethylamino)acetamido)-2-methoxybenzoic acid (13g) yield $78 \%{ }^{1} \mathrm{H}$ NMR (MeOD-d $\left.\mathrm{d}_{4}\right) \delta 7.76$ (s, 1H, ArH), 7.48 (d, J=8.5 Hz, 2H, ArH), 7.42 (d, J= $\left.9 \mathrm{~Hz}, 1 \mathrm{H}, \operatorname{ArH}\right), 7.39$ (d, J= $8.5 \mathrm{~Hz}, 2 \mathrm{H}, \mathrm{ArH}), 7.34(\mathrm{~d}, \mathrm{~J}=9 \mathrm{~Hz}, 1 \mathrm{H}, \mathrm{ArH}), 5.09\left(\mathrm{~s}, 2 \mathrm{H}, \mathrm{CH}_{2}\right), 4.08\left(\mathrm{~s}, 3 \mathrm{H}, \mathrm{CH}_{3}\right), 4.06\left(\mathrm{~s}, 2 \mathrm{H}, \mathrm{CH}_{2}\right), 3.07$ $\left(\mathrm{s}, 6 \mathrm{H}, 2 \times \mathrm{CH}_{3}\right) .{ }^{13} \mathrm{C}$ NMR $\left(\mathrm{MeOD}-\mathrm{d}_{4}\right) \delta 168.32(\mathrm{C}=\mathrm{O}), 164.38(\mathrm{C}=\mathrm{O}), 158.93(\operatorname{ArC}), 134.89(\operatorname{ArC}), 133.38$ $(\operatorname{ArC}), 133.18(\mathrm{ArCH}), 130.86(\mathrm{ArCH}), 130.64(\mathrm{ArC}), 130.29(\mathrm{ArCH}), 128.37(\mathrm{ArCH}), 122.85(\operatorname{ArC})$, $113.33(\mathrm{ArCH}), 57.94\left(\mathrm{CH}_{2}\right), 55.41\left(\mathrm{OCH}_{3}\right), 52.15\left(\mathrm{CH}_{2}\right), 43.25\left(\mathrm{CH}_{3}\right) . \mathrm{MS}$ [ESI, m/z]: calcd for $\mathrm{C}_{19} \mathrm{H}_{21} \mathrm{ClN}_{2} \mathrm{O}_{4}[\mathrm{M}+\mathrm{H}]$, 377.1268; found, 377.1279. 
Methyl 4-(N-(4-chlorobenzyl)-2-(dimethylamino)acetamido)benzoate (13h) yield $69 \%{ }^{1} \mathrm{H}$ NMR $\left(\mathrm{CDCl}_{3}\right) \delta 8.03(\mathrm{~d}, J=8.5 \mathrm{~Hz}, 2 \mathrm{H}, \mathrm{ArH}), 7.25(\mathrm{~d}, J=8.5 \mathrm{~Hz}, 2 \mathrm{H}, \mathrm{ArH}), 7.14$ (d, J=8.5 Hz, 2H, ArH), 7.08 (d, $J=8.5 \mathrm{~Hz}, 2 \mathrm{H}, \mathrm{ArH}), 4.89\left(\mathrm{~s}, 2 \mathrm{H}, \mathrm{CH}_{2}\right), 3.94\left(\mathrm{~s}, 3 \mathrm{H}, \mathrm{CH}_{3}\right), 2.89\left(\mathrm{~s}, 2 \mathrm{H}, \mathrm{CH}_{2}\right), 2.25\left(\mathrm{~s}, 6 \mathrm{H}, 2 \times \mathrm{CH}_{3}\right){ }^{13} \mathrm{C} N M R$ $\left(\mathrm{CDCl}_{3}\right) \delta 169.36(C=0), 166.13(C=0), 145.64(\operatorname{ArC}), 135.56(\operatorname{ArC}), 133.52(\operatorname{ArC}), 130.99(\operatorname{ArCH}), 130.86$ $(\mathrm{ArCH}), 130.21(\mathrm{ArC}), 128.70(\mathrm{ArCH}), 118.56(\mathrm{ArCH}), 63.67\left(\mathrm{CH}_{2}\right), 52.38\left(\mathrm{CH}_{3}\right), 52.31\left(\mathrm{CH}_{2}\right), 45.54$ $\left(2 \times \mathrm{CH}_{3}\right) . \mathrm{MS}[\mathrm{ESI}, \mathrm{m} / \mathrm{z}]$ : calcd for $\mathrm{C}_{19} \mathrm{H}_{21} \mathrm{ClN}_{2} \mathrm{O}_{3}[\mathrm{M}+\mathrm{H}], 361.1319$; found, 361.1 .

$\mathbf{N}$-(4-chlorobenzyl)- $\mathbf{N}$-(2-cyanophenyl)-2-(dimethylamino)acetamide (13i) yield $71 \%{ }^{1} \mathrm{H}$ NMR $\left(\mathrm{CDCl}_{3}\right) \delta 7.74(\mathrm{~d}, J=8 \mathrm{~Hz}, 1 \mathrm{H}, \mathrm{ArH}), 7.54(\mathrm{t}, J=7.5 \mathrm{~Hz}, 1 \mathrm{H}, \operatorname{ArH}), 7.46(\mathrm{t}, J=7.5 \mathrm{~Hz}, 1 \mathrm{H}, \operatorname{ArH}), 7.24$ (d, J= $8.5 \mathrm{~Hz}, 2 \mathrm{H}, \operatorname{ArH}), 7.14(\mathrm{~d}, J=8.5 \mathrm{~Hz}, 2 \mathrm{H}, \operatorname{ArH}), 6.92(\mathrm{~d}, J=8 \mathrm{~Hz}, 1 \mathrm{H}, \operatorname{ArH}), 4.48(\mathrm{~d}, J=14.5 \mathrm{~Hz}, 1 \mathrm{H}, \mathrm{CH}$ ), $4.38(\mathrm{~d}, J=14.5 \mathrm{~Hz}, 1 \mathrm{H}, \mathrm{CH}), 2.87\left(\mathrm{~s}, 2 \mathrm{H}, \mathrm{CH}_{2}\right), 2.20\left(\mathrm{~s}, 6 \mathrm{H}, 2 \mathrm{XCH}_{3}\right) .{ }^{13} \mathrm{CNMR}\left(\mathrm{CDCl}_{3}\right) \delta 169.41(\mathrm{C}=\mathrm{O})$, $143.86(\mathrm{CN}), 134.84(\mathrm{ArC}), 133.84(\mathrm{ArCH}), 133.80(\mathrm{ArC}), 133.61(\mathrm{ArCH}), 130.74(\mathrm{ArCH}), 130.35(\mathrm{ArCH})$, $128.72(2 \mathrm{ArCH}), 115.93(\mathrm{ArC}), 113.57(\mathrm{ArC}), 61.28\left(\mathrm{CH}_{2}\right), 51.98\left(\mathrm{CH}_{2}\right), 45.29\left(2 \times \mathrm{CH}_{3}\right) . \mathrm{MS}[\mathrm{ESI}, \mathrm{m} / \mathrm{z}]$ : calcd for $\mathrm{C}_{18} \mathrm{H}_{18} \mathrm{ClN}_{3} \mathrm{O}[\mathrm{M}+\mathrm{H}], 328.1212$; found, 328.1217.

$\mathrm{N}$-(4-chlorobenzyl)-N-(3-cyanophenyl)-2-(dimethylamino)acetamide (13j) yield $96 \%{ }^{1} \mathrm{H} N M R$ $\left(\mathrm{CDCl}_{3}\right) \delta 7.64(\mathrm{~d}, J=7.5 \mathrm{~Hz}, 1 \mathrm{H}, \operatorname{ArH}), 7.48(\mathrm{t}, J=8 \mathrm{~Hz}, 1 \mathrm{H}, \operatorname{ArH}), 7.40(\mathrm{~s}, 1 \mathrm{H}, \operatorname{ArH}), 7.28(\mathrm{~d}, J=8 \mathrm{~Hz}$, $2 \mathrm{H}, \mathrm{ArH}), 7.14(\mathrm{~d}, \mathrm{~J}=8.5 \mathrm{~Hz}, 2 \mathrm{H}, \mathrm{ArH}), 4.88\left(\mathrm{~s}, 2 \mathrm{H}, \mathrm{CH}_{2}\right), 2.87\left(\mathrm{~s}, 2 \mathrm{H}, \mathrm{CH}_{2}\right), 2.25\left(\mathrm{~s}, 6 \mathrm{H}, 2 \times \mathrm{CH}_{3}\right) ;{ }^{13} \mathrm{C} \mathrm{NMR}$ $\left(\mathrm{CDCl}_{3}\right) \delta 169.36(\mathrm{C}=\mathrm{O}), 142.50(\mathrm{ArC}), 135.17(\mathrm{ArC}), 133.72(\mathrm{ArC}), 133.18(\mathrm{ArCH}), 131.95(\mathrm{ArCH})$, $131.73(\mathrm{ArCH}), 130.51(\mathrm{ArCH}), 130.11(\mathrm{ArCH}), 128.86(\mathrm{ArCH}), 117.61(\mathrm{CN}), 113.72(\mathrm{ArC}), 61.14\left(\mathrm{CH}_{2}\right)$, $52.41\left(\mathrm{CH}_{2}\right), 45.44\left(2 \times \mathrm{CH}_{3}\right)$. MS [ESI, m/z]: calcd for $\mathrm{C}_{18} \mathrm{H}_{18} \mathrm{ClN}_{3} \mathrm{O}[\mathrm{M}+\mathrm{H}]$, 328.1217; found, 328.1233.

$\mathrm{N}$-(4-chlorobenzyl)-N-(4-cyanophenyl)-2-(dimethylamino)acetamide (13k) yield $87 \%{ }^{1} \mathrm{H} N M R$ $\left(\mathrm{CDCl}_{3}\right) \delta 7.67(\mathrm{~d}, J=8.5 \mathrm{~Hz}, 2 \mathrm{H}, \operatorname{ArH}), 7.26(\mathrm{~d}, J=8.5 \mathrm{~Hz}, 2 \mathrm{H}, \operatorname{ArH}), 7.17(\mathrm{~d}, J=8.5 \mathrm{~Hz}, 2 \mathrm{H}, \operatorname{ArH}), 7.13$ $(\mathrm{d}, J=8.5 \mathrm{~Hz}, 2 \mathrm{H}, \mathrm{ArH}), 4.90\left(\mathrm{~s}, 2 \mathrm{H}, \mathrm{CH}_{2}\right), 2.90\left(\mathrm{~s}, 2 \mathrm{H}, \mathrm{CH}_{2}\right), 2.23\left(\mathrm{~s}, 6 \mathrm{H}, 2 \times \mathrm{CH}_{3}\right) ;{ }^{13} \mathrm{CNMR}\left(\mathrm{CDCl}_{3}\right) \delta$ $169.28(C=0), 145.75(\operatorname{ArC}), 135.23(\operatorname{ArC}), 133.73(\operatorname{ArC}), 133.45(\mathrm{ArCH}), 129.97(\mathrm{ArCH}), 129.18(\operatorname{ArCH})$, $128.86(\mathrm{ArCH}), 117.91(\mathrm{CN}), 111.93(\mathrm{ArC}), 61.31\left(\mathrm{CH}_{2}\right), 52.35\left(\mathrm{CH}_{2}\right), 45.45\left(2 \times \mathrm{CH}_{3}\right) . \mathrm{MS}$ [ESI, m/z]: $328.12[\mathrm{M}+\mathrm{H}], 350.10[\mathrm{M}+\mathrm{Na}]$, anal. calcd for $\mathrm{C}_{18} \mathrm{H}_{18} \mathrm{ClN}_{3} \mathrm{O}: \mathrm{C}, 65.95 ; \mathrm{H}, 5.53 ; \mathrm{N}, 12.81$. Found: $\mathrm{C}$, $65.85 ; H, 5.60 ; \mathrm{N}, 12.72$.

$\mathbf{N}$-(3-cyanophenyl)-2-(dimethylamino)- $\mathbf{N}$-(4-fluorobenzyl)acetamide (13I) yield $65 \%$ \% ${ }^{1} \mathrm{H}$ NMR $\left(\mathrm{CDCl}_{3}\right) \delta 7.63$ (d, J=7.5 Hz, 1H, ArH), $7.48(\mathrm{t}, J=7.5 \mathrm{~Hz}, 1 \mathrm{H}, \mathrm{ArH}), 7.37$ (s, 1H, ArH), 7.24 (d, J=7 Hz, 1H, $\operatorname{ArH}), 7.16(\mathrm{dd}, J=9,5.5 \mathrm{~Hz}, 2 \mathrm{H}, \mathrm{ArH}), 6.99(\mathrm{t}, \mathrm{J}=8.5 \mathrm{~Hz}, 2 \mathrm{H}, \mathrm{ArH}), 4.88\left(\mathrm{~s}, 2 \mathrm{H}, \mathrm{CH}_{2}\right), 2.84\left(\mathrm{~s}, 2 \mathrm{H}, \mathrm{CH}_{2}\right)$, $2.24\left(\mathrm{~s}, 6 \mathrm{H}, 2 \times \mathrm{CH}_{3}\right) \cdot{ }^{13} \mathrm{C} \mathrm{NMR}\left(\mathrm{CDCl}_{3}\right) \delta 165.56(\mathrm{C}=\mathrm{O}), 162.36\left(\mathrm{~d},{ }^{1} \mathrm{~J}_{\mathrm{C}-\mathrm{F}}=245 \mathrm{~Hz}, \operatorname{ArC}\right), 142.54(\operatorname{ArC})$, $133.21(\mathrm{ArCH}), 132.49(\mathrm{ArC}), 132.07(\mathrm{ArCH}), 131.67(\mathrm{ArCH}), 130.63(\mathrm{ArCH}), 130.45(\mathrm{ArCH}), 117.62$ $(\mathrm{CN}), 115.60\left(\mathrm{~d},{ }^{2} \mathrm{~J}_{\mathrm{C}-\mathrm{F}}=21.5 \mathrm{~Hz}, \operatorname{ArCH}\right), 113.70(\mathrm{ArC}), 61.20\left(\mathrm{CH}_{2}\right), 52.35\left(\mathrm{CH}_{2}\right), 45.47\left(2 \times \mathrm{CH}_{3}\right) . \mathrm{MS}[\mathrm{ESI}$, $\mathrm{m} / \mathrm{z}$ ]: calcd for $\mathrm{C}_{18} \mathrm{H}_{18} \mathrm{FN}_{3} \mathrm{O}[\mathrm{M}+\mathrm{H}]$, 312.1507; found, 312.1506. 
5-(N-(4-chlorobenzyl)-2-(dimethylamino)acetamido)-2-hydroxybenzoic acid (13m) yield $97 \%$. The mixture of $(13 \mathrm{c} ; 1.5 \mathrm{mmol})$ in $\mathrm{MeOH}$ and aq. $1 \mathrm{M} \mathrm{NaOH}(0.7 \mathrm{~mL})$ solution was stirred at $60^{\circ}$ for $24 \mathrm{~h}$. The mixture was cooled and $1 \mathrm{M} \mathrm{HCl}$ was added for neutralization. The white product precipitated and recrystalised from MeOH. ${ }^{1} \mathrm{H}$ NMR $\left(\mathrm{MeOH}-\mathrm{d}_{4}\right) \delta 7.55(\mathrm{~d}, \mathrm{~J}=3 \mathrm{~Hz}, 1 \mathrm{H}, \mathrm{ArH}), 7.31(\mathrm{~d}, J=8.5 \mathrm{~Hz}, 2 \mathrm{H}$, $\operatorname{ArH}), 7.23(\mathrm{~d}, J=8.5 \mathrm{~Hz}, 2 \mathrm{H}, \operatorname{ArH}), 7.00(\mathrm{dd}, J=8.5,3 \mathrm{~Hz}, 1 \mathrm{H}, \operatorname{ArH}), 6.83(\mathrm{~d}, J=8.5 \mathrm{~Hz}, 1 \mathrm{H}, \operatorname{ArH}), 4.90(\mathrm{~s}$, $\left.2 \mathrm{H}, \mathrm{CH}_{2}\right), 3.87\left(\mathrm{~s}, 2 \mathrm{H}, \mathrm{CH}_{2}\right), 2.88\left(\mathrm{~s}, 6 \mathrm{H}, 2 \times \mathrm{CH}_{3}\right) .{ }^{13} \mathrm{C} \mathrm{NMR}\left(\mathrm{MeOH}-\mathrm{d}_{4}\right) 173.11(\mathrm{C}=\mathrm{O}), 164.58(\mathrm{C}=\mathrm{O})$, $162.03(\operatorname{ArC}), 135.16(\operatorname{ArC}), 133.31(\operatorname{ArC}), 132.03(\mathrm{ArCH}), 130.26(\mathrm{ArCH}), 130.08(\mathrm{ArCH}), 128.47(\operatorname{ArC})$, 128.30 ( $\mathrm{ArCH}), 119.97(\mathrm{ArC}), 117.58(\mathrm{ArCH}), 58.01\left(\mathrm{CH}_{2}\right), 52.27\left(\mathrm{CH}_{2}\right), 43.08\left(2 \times \mathrm{CH}_{3}\right) . \mathrm{MS}[\mathrm{ESI}, \mathrm{m} / \mathrm{z}]$ : calcd for $\mathrm{C}_{18} \mathrm{H}_{19} \mathrm{ClN}_{2} \mathrm{O}_{4}[\mathrm{M}+\mathrm{H}]$, 363.1112; found, 363.1127.

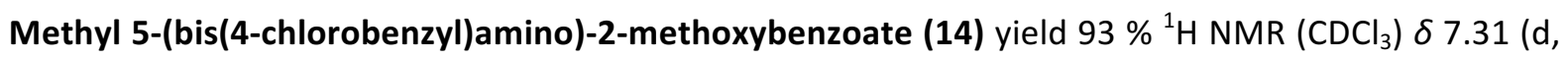
$J=8.5 \mathrm{~Hz}, 4 \mathrm{H}, \operatorname{ArH}), 7.26(\mathrm{~d}, J=2.5 \mathrm{~Hz}, 1 \mathrm{H}, \operatorname{ArH}), 7.18(\mathrm{~d}, J=9 \mathrm{~Hz}, 4 \mathrm{H}, \operatorname{ArH}), 6.85(\mathrm{~d}, J=9 \mathrm{~Hz}, 1 \mathrm{H}, \operatorname{Ar} H)$, $6.82(\mathrm{dd}, \mathrm{J}=9,3 \mathrm{~Hz}, 1 \mathrm{H}, \mathrm{ArH}), 4.50\left(\mathrm{~s}, 4 \mathrm{H}, 2 \times \mathrm{CH}_{2}\right), 3.87\left(\mathrm{~s}, 3 \mathrm{H}, \mathrm{CH}_{3}\right), 3.84\left(\mathrm{~s}, 3 \mathrm{H}, \mathrm{CH}_{3}\right) ;{ }^{13} \mathrm{CNMR}\left(\mathrm{CDCl}_{3}\right)$ $\delta 167.02(C=0), 151.79(\operatorname{ArC}), 142.68(\operatorname{ArC}), 136.67(\operatorname{ArC}), 132.84(\operatorname{ArC}), 128.80(\operatorname{ArCH}), 128.50(\operatorname{ArCH})$, $120.81(\mathrm{ArC}), 119.27(\mathrm{ArCH}), 117.07(\mathrm{ArCH}), 114.05(\mathrm{ArCH}), 56.76\left(\mathrm{CH}_{3}\right), 54.60\left(\mathrm{CH}_{2}\right), 52.12\left(\mathrm{CH}_{3}\right) . \mathrm{MS}$ [ESI, $\mathrm{m} / \mathrm{z}]: 430.12[\mathrm{M}+\mathrm{H}]$. calcd for $\mathrm{C}_{23} \mathrm{H}_{21} \mathrm{Cl}_{2} \mathrm{NO}_{3}[\mathrm{M}+\mathrm{H}], 430.0971$; found, 430.0972 .

5-(Bis(4-chlorobenzyl)amino)-2-methoxybenzoic acid (15) yield $75 \% .{ }^{1} \mathrm{H} N M R\left(\mathrm{CDCl}_{3}\right) \delta 9.57$ (bs, $1 \mathrm{H}$, $\mathrm{COOH}), 7.61(\mathrm{~d}, J=3 \mathrm{~Hz}, 4 \mathrm{H}, \operatorname{ArH}), 7.28(\mathrm{~d}, J=8.5 \mathrm{~Hz}, 1 \mathrm{H}, \operatorname{ArH}), 7.16$ (d, J=8.5 Hz, 4H, ArH), 6.91 (d, J=9

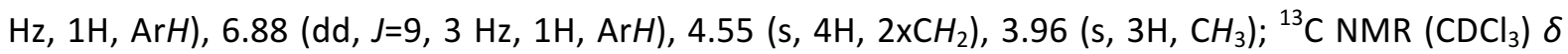
$165.95(C=0), 150.46(\operatorname{ArC}), 143.85(\operatorname{ArC}), 136.40(\operatorname{ArC}), 132.89(\operatorname{ArC}), 128.87(\operatorname{ArCH}), 128.38(\operatorname{ArCH})$, $120.04(\mathrm{ArCH}), 118.19(\mathrm{ArC}), 117.60(\mathrm{ArCH}), 113.37(\mathrm{ArCH}), 57.13\left(\mathrm{CH}_{3}\right), 54.39\left(\mathrm{CH}_{2}\right)$. calcd for $\mathrm{C}_{22} \mathrm{H}_{19} \mathrm{Cl}_{2} \mathrm{NO}_{3}[\mathrm{M}-\mathrm{H}]$, 414.0669; found, 414.0664 . 


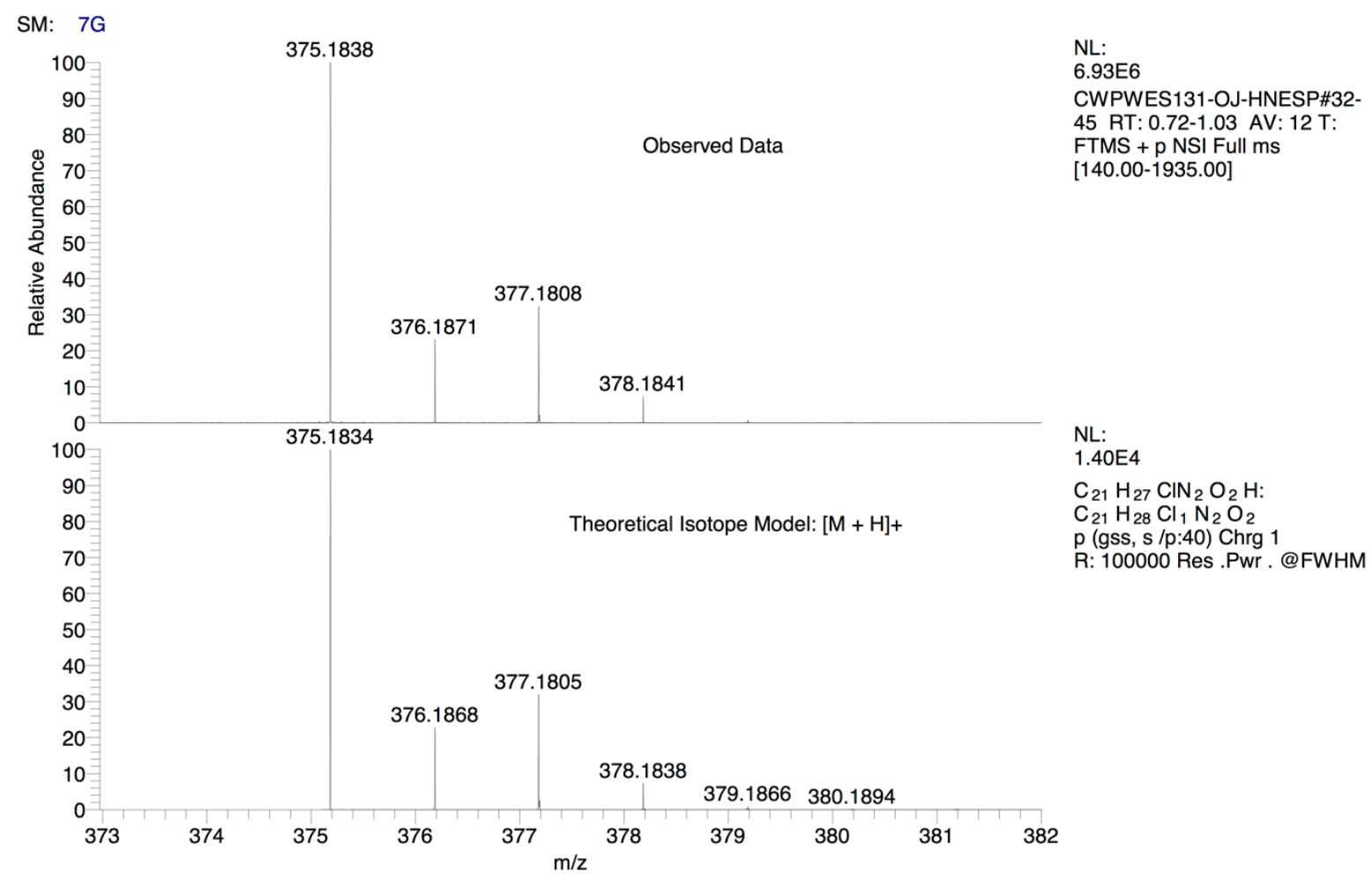

Figure 4. Mass spectrum of compound 5c

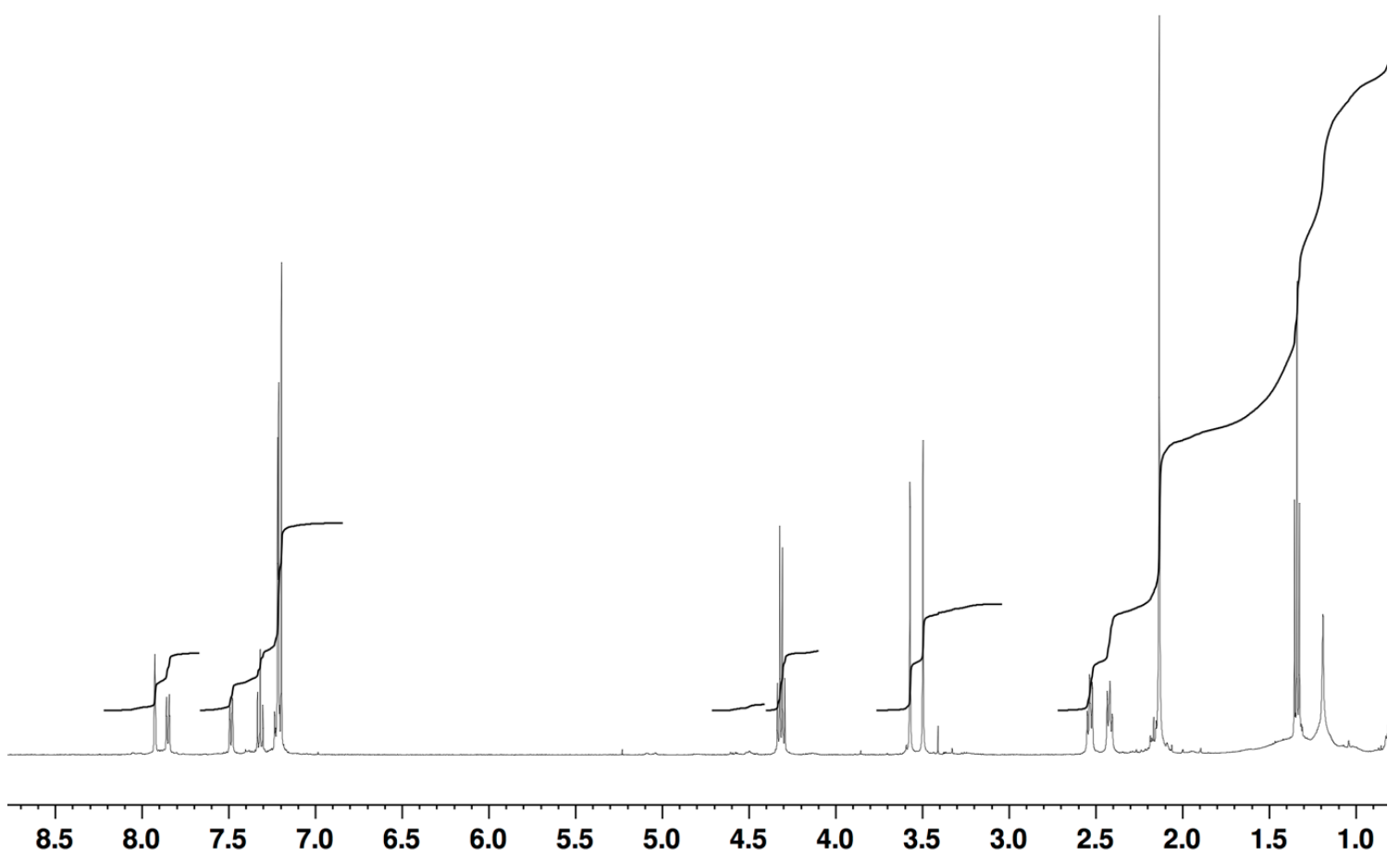

Figure 5. ${ }^{1} \mathrm{H}$ NMR spectrum of compound $5 \mathrm{c}$ 


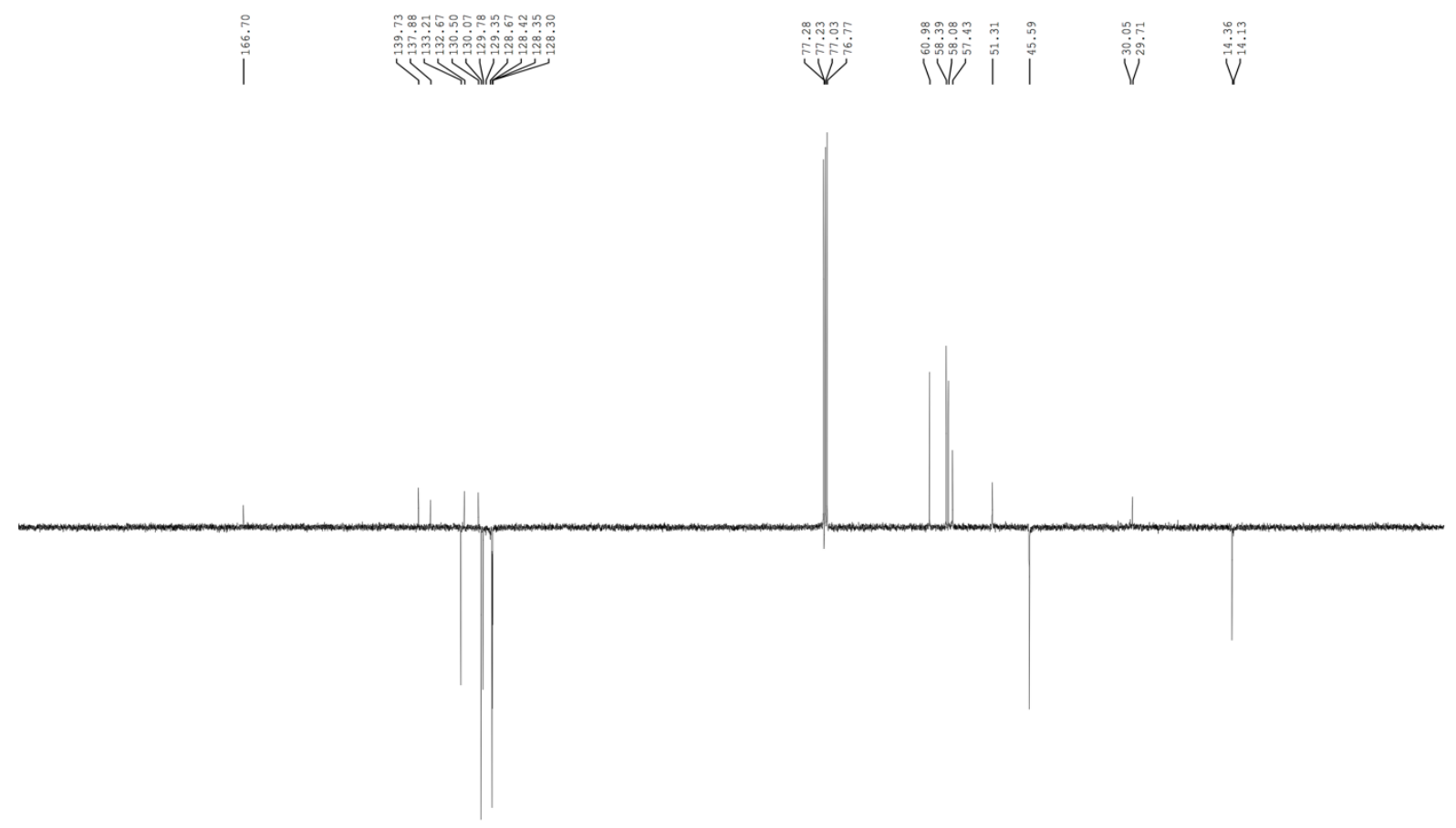

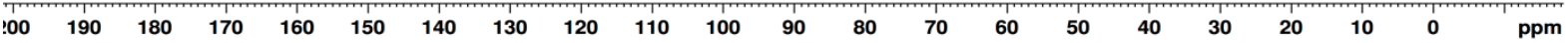

Figure 6. ${ }^{13} \mathrm{C}$ NMR spectrum of compound $5 \mathrm{c}$ 